\title{
AMUSE-Field I: NUCLEAR X-RAY PROPERTIES OF LOCAL FIELD AND GROUP SPHEROIDS ACROSS THE STELLAR MASS SCALE
}

\author{
Brendan Miller $^{1}$, Elena Gallo ${ }^{1}$, Tommaso Treu ${ }^{2}$, and Jong-HaK Woo $^{3}$ \\ ${ }^{1}$ Department of Astronomy, University of Michigan, Ann Arbor, MI 48109, USA \\ 2 Physics Department, University of California, Santa Barbara, CA 93106, USA \\ ${ }^{3}$ Astronomy Program, Department of Physics and Astronomy, Seoul National University, Seoul, Republic of Korea \\ Received 2011 August 20; accepted 2011 December 19; published 2012 February 14
}

\begin{abstract}
We present the first results from AMUSE-Field, a Chandra survey designed to characterize the occurrence and intensity of low-level accretion onto supermassive black holes (SMBHs) at the center of local early-type field galaxies. This is accomplished by means of a Large Program targeting a distance-limited ( $<30 \mathrm{Mpc})$ sample of 103 early types spanning a wide range in stellar masses. We acquired new ACIS-S observations for 61 objects down to a limiting (0.3-10 keV) luminosity of $2.5 \times 10^{38} \mathrm{erg} \mathrm{s}^{-1}$, and we include an additional 42 objects with archival (typically deeper) coverage. A nuclear X-ray source is detected in 52 out of the 103 galaxies. After accounting for potential contamination from low-mass X-ray binaries, we estimate that the fraction of accreting SMBHs within the sample is $45 \% \pm 7 \%$, which sets a firm lower limit on the occupation fraction within the field. The measured nuclear X-ray luminosities are invariably highly sub-Eddington, with $L_{\mathrm{X}} / L_{\mathrm{Edd}}$ ratios between $\sim 10^{-4}$ and $10^{-8}$. As also found in a companion survey targeting Virgo early types, the active fraction increases with increasing host galaxy stellar mass, reflective of "Eddington incompleteness" within the lower-mass objects. For the Field sample, the average nuclear X-ray luminosity scales with the host stellar mass as $M_{\mathrm{star}}^{0.71 \pm 0.10}$, with an intrinsic scatter of $0.73 \pm 0.09$ dex. Qualitatively similar results hold for morphologically homogeneous (type E) or uniform sensitivity (new observations only) subsets. A majority of the AMUSE-Field galaxies (78\%) inhabit groups, enabling us to investigate the influence of group richness on nuclear activity. We see no evidence for a positive correlation between nuclear X-ray luminosity, normalized to host properties, and galaxy density. Rather, while the scatter is substantial, it appears that the Eddington-scaled X-ray luminosity of group members may be slightly lower than for isolated galaxies, and that this trend continues to cluster early types.
\end{abstract}

Key words: black hole physics - galaxies: nuclei

Online-only material: color figures

\section{INTRODUCTION}

Convincing evidence that supermassive black holes (SMBHs) are able to form in the early universe is provided by observations of high-redshift quasars (e.g., Volonteri \& Rees 2006; Vestergaard \& Osmer 2009; Willott et al. 2010; Treister et al. 2011). The seeds for these early SMBHs may have been produced from supermassive (e.g., Begelman 2010) or Population III stars, or from direct gas collapse (e.g., Volonteri \& Natarajan 2009), perhaps in massive protogalaxy mergers (e.g., Mayer et al. 2010; Volonteri 2010). While high-redshift quasars generally display optical/UV and X-ray properties similar to those of their local analogues (e.g., Shemmer et al. 2006), Spitzer observations indicate that many are young sources (some lack hot dust) that are growing rapidly (Jiang et al. 2010). In contrast, similarly massive SMBHs at more moderate redshifts are growing more slowly (e.g., Netzer et al. 2007). Indeed, the growth of SMBHs appears to be "anti-hierarchical" in the sense that active accretion is concentrated in higher/lower-mass SMBHs at earlier/later cosmological times (e.g., Heckman et al. 2004; Merloni \& Heinz 2007; Shankar et al. 2009; Gallo et al. 2010; Goulding et al. 2010; Kelly et al. 2010; Lamastra et al. 2010; Schulze \& Wisotzki 2010; Schawinski et al. 2010).

The peak of the quasar space density around $z \sim 2$ (e.g., Brown et al. 2006; Hopkins et al. 2007; Kelly et al. 2010) ${ }^{4}$

\footnotetext{
4 The quasar peak redshift is luminosity dependent, displaying a similar "downsizing" effect (e.g., Hasinger et al. 2005; Croom et al. 2009) to that found for SMBH growth.
}

and the $\sim 10^{8}$ yr quasar lifetime (e.g., Yu \& Tremaine 2002) suggest (e.g., Soltan 1982) that "inactive" galactic nuclei typically also host SMBHs accreting at low levels and/or radiating inefficiently in a post-quasar stage that may result after much of the available fuel has been consumed or expelled (e.g., Hopkins et al. 2005). The distinctions between active galactic nuclei (AGNs), low-luminosity AGNs (Ho 1999, 2008), and formally inactive galaxies are somewhat arbitrary, but as a general guideline (which we will adhere to in this work) AGNs display bolometric, Eddington-scaled luminosities in excess of a few percent, low-luminosity AGNs are in the range $10^{-4}<L / L_{\text {Edd }}<10^{-2}$, and inactive nuclei are highly subEddington, with $L / L_{\text {Edd }} \lesssim 10^{-4}$. The Milky Way is one example of a formally inactive galaxy. It is known to host a quiescent central SMBH with mass of $3.6 \times 10^{6} M_{\odot}$ (e.g., Schödel et al. 2009) from which low-level, persistent accretionpowered X-ray emission has been detected $\left(L_{\mathrm{X}} / L_{\mathrm{Edd}} \simeq\right.$ $10^{-11}$; Baganoff et al. 2001). Even low levels of SMBH accretion-powered activity in nearby galaxies may be detected efficiently in sensitive, high spatial resolution X-ray observations, although contamination from bright X-ray binaries must be properly accounted for when dealing with nuclear $\mathrm{X}$-ray luminosities comparable to the Eddington limit for a few solar masses (e.g., Zhang et al. 2009; Gallo et al. 2010). This problem can be substantially alleviated by restricting the search for highly sub-Eddington nuclei to early-type galaxies, as they conveniently avoid the brighter high-mass X-ray binaries produced concurrently with star formation (e.g., King et al. 2001; Ghosh et al. 2009). 
From X-ray and other observations as well as numerical simulations, a coherent scenario for the history and observed characteristics of quiescent or weakly accreting SMBHs in early-type galaxies is emerging. Although hot halo gas or stellar winds provide readily available fuel and gas infall may proceed at near the Bondi rate, outflows can drive off a substantial fraction of the accreting mass and the radiative efficiency is inferred from observed spectral energy distributions to be highly sub-Eddington (e.g., Di Matteo et al. 2000; Pellegrini 2005; Soria et al. 2006b).

The growth of SMBHs is believed to be closely tied to the evolution of their hosts (as evidenced by, for example, the correlations between SMBH mass and bulge luminosity or central stellar velocity dispersion; see, e.g., overview by Ferrarese $\&$ Ford 2005). Radiative and mechanical AGN feedback acts to regulate SMBH accretion and quench star formation (e.g., Ciotti et al. 2009, 2010; Pellegrini et al. 2012), and mechanical feedback continues to play an important role in low-activity "radio-mode" systems (e.g., Croton et al. 2006; Merloni \& Heinz 2007; Marulli et al. 2008). The assembly of early-type galaxies apparently proceeds hierarchically, despite the older ages and shorter star formation timescales of massive ellipticals (e.g., De Lucia et al. 2006; Eliche-Moral et al. 2010), with cold-gas-rich/poor ("wet"/"dry") mergers tending to produce disky/boxy types (e.g., Kang et al. 2007; Hopkins \& Quataert 2011).

Naturally the properties of higher-mass SMBHs (in more massive galaxies) may be more easily constrained; the dominance of compact stellar nuclei at lower luminosities (e.g., Ferrarese et al. 2006a) presented initial observational challenges to establishing whether lower-mass early-type galaxies necessarily even contained an SMBH. The presence of "missing light" or cores within luminous ellipticals may be explained by binary black holes disrupting the interior region in the process of merging (e.g., Kormendy et al. 2009, and references therein), and so preserved nuclear star clusters could potentially indicate a lack of an SMBH binary phase (e.g., Kang et al. 2007). However, it is now clear that SMBHs may coexist with nuclear star clusters (e.g., Seth et al. 2008; Graham \& Spitler 2009; Kormendy et al. 2009).

To quantify the rate of low-level SMBH activity over a well-defined sample of nearby early-type galaxies, our group carried out the AGN MUltiwavelength Survey of Early-Type Galaxies in the Virgo cluster. AMUSE-Virgo (ID 8900784, ChandraCycle 8, PI: Treu, $454 \mathrm{ks}$ ) targeted the 100 spheroidal galaxies that compose the Hubble Space Telescope (HST) Advanced Camera for Surveys (ACS) Virgo Cluster Survey (VCS; Côté et al. 2004) with Chandra ACIS-S and Spitzer MIPS (Gallo et al. 2008, 2010, hereafter G08, G10; Leipski et al. 2012). The VCS sample was selected based solely on optical properties and spans a wide range in stellar mass, from $10^{8}$ to $10^{12} M_{\odot}$. At the average Virgo distance of $16.5 \mathrm{Mpc}$ (Mei et al. 2007), the AMUSE-Virgo Chandra snapshot observations (about $5 \mathrm{ks}$ each) reached a $2 \sigma$ limiting $0.5-7 \mathrm{keV}$ luminosity of $1.3 \times 10^{38} \mathrm{erg} \mathrm{s}^{-1}$ (i.e., close to the Eddington limit for $\left.1 M_{\odot}\right)$. A nuclear X-ray source was detected by G10 in 32/ 100 objects, which, after taking into consideration the minor but non-negligible effects of low-mass X-ray binary (LMXB) contamination, sets a lower limit of $24 \%-34 \%$ (at the $95 \%$ confidence level) to the occupation fraction of SMBHs in the nuclei of cluster early-type galaxies. Within the AMUSE-Virgo survey the fraction of X-ray-active $\mathrm{SMBHs}$ is seen to increase with host stellar mass, ${ }^{5}$ but in a manner consistent with arising from "Eddington incompleteness," i.e., the intrinsic inability of any luminosity-limited survey to reach the same Eddingtonscaled luminosity level across a wide range of inferred black hole masses. Despite this effect, which obviously penalizes lower mass black holes, the average Eddington-scaled X-ray luminosity of the AMUSE-Virgo galaxies is found to scale inversely with black hole mass as $\left\langle L_{\mathrm{X}} / L_{\mathrm{Edd}}\right\rangle \propto M_{\mathrm{BH}}^{-0.62}$, so that nearby lower-mass SMBHs are relatively more X-ray active (a trend G10 refers to as "downsizing in black hole accretion"). In all cases the X-ray luminosity is highly sub-Eddington, with $10^{-8} \lesssim L_{\mathrm{X}} / L_{\text {Edd }} \lesssim 10^{-5}$.

While this and other studies (e.g., Pellegrini 2005; Zhang et al. 2009; Boroson et al. 2011) have provided useful and detailed census of low-level SMBH activity within the local universe, including as a function of the host galaxy properties (Pellegrini 2010), the impact of environment on the occurrence and intensity of SMBH activity remains unclear. The properties of early-type galaxies in more sparsely populated regions are distinct from their counterparts in clusters: relative to cluster sources, field early-type galaxies face reduced ram pressure stripping (e.g., Acreman et al. 2003; Gavazzi et al. 2010; Shin et al. 2012) and on average contain more cold gas and tend to have younger stellar populations (e.g., Thomas et al. 2005; Oosterloo et al. 2010). Within clusters, tidal interactions play an important role (e.g., Gnedin 2003), for example, as harassment from highspeed encounters (e.g., Treu et al. 2003); such effects are only infrequently relevant to isolated field galaxies, although within groups the lower galaxy velocity dispersions can help facilitate mergers. Early work with ROSAT links the environment with $\mathrm{X}$-ray emission, but to a debated degree, for example, Brown \& Bregman (2000) found that cluster early-type galaxies tend to be more X-ray luminous than their field counterparts and hypothesized that this results from environmental suppression of outflows or the availability for accretion of hot intergalactic gas within clusters, whereas O'Sullivan et al. (2001) found similar $L_{\mathrm{X}}\left(L_{B}\right)$ relations for cluster, group, and field galaxies. While such results are intriguing, their utility is necessarily restricted by the limited angular resolution of ROSAT, which makes it difficult to disentangle diffuse thermal X-ray or off-nuclear $\mathrm{X}$-ray binary emission from that linked to the SMBH.

The goal of this work is to investigate low-level SMBH activity within a well-defined sample of nearby field spheroidal galaxies (E and E-S0 galaxies) and to characterize the dependence (if any) of such activity on environmental effects, such as living in isolation versus belonging to a group of galaxies, or belonging to a poor versus a rich group. Toward this end, we were awarded a Large Chandra program building on and extending AMUSE-Virgo to non-cluster environments: AMUSEField (ID 11620915, Chandra Cycle 11, 479 ks, PI: Gallo) carried out new observations of 61 field early-type galaxies located within $30 \mathrm{Mpc}$ and spanning a wide range of stellar and black hole masses. The new observations are supplemented with an additional 42 objects with archival coverage, for a total of 103 objects.

This paper is structured as follows: Section 2 describes the sample selection and Chandra data reduction; Section 3 provides the X-ray results; Section 4 investigates the dependence of nuclear X-ray luminosity on stellar mass; Section 5 discusses

\footnotetext{
5 That SMBH activity in general is detected more frequently with larger host stellar mass or luminosity is known since the first Palomar sample (e.g., Ho et al. 1997).
} 
environmental influences on SMBH accretion; Section 6 summarizes and concludes. $L_{\mathrm{X}}, M_{\mathrm{star}}$, and $M_{\mathrm{BH}}$ are the nuclear 0.3-10 keV X-ray luminosity, galaxy stellar mass, and black hole mass, respectively, and are given in units of $\mathrm{erg} \mathrm{s}^{-1}, M_{\odot}$, and $M_{\odot}$ throughout. Errors are $1 \sigma$ unless otherwise noted.

\section{SAMPLE SELECTION AND Chandra DATA REDUCTION}

The AMUSE-Field sample was selected based only on optical galaxy properties, in the following manner. We first searched the HyperLeda ${ }^{6}$ catalog (Paturel et al. 2003) for spheroidal galaxies, conservatively defined as having type E or E-S0, with inferred heliocentric velocities less than $1800 \mathrm{~km} \mathrm{~s}^{-1}$ and distances less than $30 \mathrm{Mpc}^{7}$ Objects near the Galactic plane $\left(|b|<30^{\circ}\right)$ were excluded, to avoid absorption effects. Objects near the Virgo cluster (within $\simeq 6^{\circ}$ of $12.44 \mathrm{hr}, 12.72$ ) or the Fornax cluster (within $\simeq 0.2$ of $3.64 \mathrm{hr},-35.45$ ) were likewise excluded, to avoid contamination by cluster galaxies; these are the only clusters lying within the considered volume. Finally, objects were required to have absolute $B$ magnitudes more luminous than $M_{B}=-13$. This results in a list of 204 objects. Of these 204 objects, 42 already possessed high-quality archival Chandra ACIS-S3 imaging observations, which we define here as exposure $>3 \mathrm{ks}$ and off-axis angle $<5^{\prime}$. Sixtyone additional targets were selected such that the full AMUSEField sample, made up of 103 early-type galaxies, provides representative coverage of a wide range in absolute $B$ magnitude. Chandra exposures for the 61 new targets (which we shall generally refer to as "snapshots") vary between $\sim 2$ and $12 \mathrm{ks}$ and were chosen to achieve a common sensitivity threshold of $2.5 \times 10^{38} \mathrm{erg} \mathrm{s}^{-1}(0.3-10 \mathrm{keV})$. Optical properties of the individual galaxies ${ }^{8}$ are listed in Table 1, along with their derived stellar and black hole masses (Section 4.1) and their group membership (Section 5).

The Chandra ACIS-S X-ray data were reduced in a uniform fashion following the general procedure applied to the AMUSE-Virgo sample (G08, G10). Here, we describe the astrometry correction, event reprocessing, light-curve filtering, source detection, and aperture photometry. The calculation of X-ray luminosities from net count rates is described in Section 3.1.

The default Chandra astrometry is generally accurate to $\lesssim 0^{\prime \prime} .3$ ( $1 \sigma$; e.g., Section 3 of Eckart et al. 2005), which is sufficient to identify uniquely which X-ray source, if any, is located nearest the galactic nucleus. For cases in which the pointing fell within the SDSS footprint, we improve the X-ray astrometry through cross-matching X-ray sources to their optical counterparts. A first-pass X-ray source list was created by running wavdetect on a $0.3-7 \mathrm{keV}$ image generated from the pipeline level 2 event file. For each non-nuclear $\left(>10^{\prime \prime}\right)$ ACIS-S3 X-ray source, the nearest primary SDSS object with $r$-band magnitude $m_{r}<23$ (hence highly accurate optical position) was sought, to within a radius dependent on the wavdetect estimated net counts as well as the $\mathrm{X}$-ray off-axis angle (this X-ray positional uncertainty is based

\footnotetext{
6 http://leda.univ-lyon1.fr/

7 For significantly larger distances, the total stellar mass enclosed by the Chandra point-spread function becomes sufficiently large that the possibility of cumulative LMXB contamination is non-negligible; see discussion in G10. 8 The full AMUSE-Field sample includes four objects for which we had X-ray data in hand but that do not technically meet all selection criteria. NGC 5077 has a distance of $40.2 \mathrm{Mpc}$ but is otherwise suitable. PGC 132768, 042737, and 042596 (all X-ray non-detections) do not have a morphological type listed in HyperLeda; digital sky images suggest that their morphologies, while spheroidal, are somewhat irregular.
}

on Section 2.3 of Hong et al. 2005). The average offset in right ascension and declination was calculated using weighting by the inverse uncertainties; any $3 \sigma$ outliers were discarded and this process was repeated. The resulting offsets were converted to pixel shifts and used to generate a new aspect solution file. The number of "clean" matches to SDSS objects used to calculate the shifts ranged from 2 to 10 , and in no cases were the shifts in $x$ or $y$ greater than one pixel (i.e., 0.'5). Astrometry for pointings outside the SDSS footprint, or for observations lacking sufficient high-confidence cross matches, was not adjusted.

A background light curve of the S3 chip (with point sources excluded), binned to $200 \mathrm{~s}$, was screened for flaring using the deflare script. Periods of anomalously high or low background were identified as any $>3 \sigma$ deviations from the mean count rate for most observations, and as any $>2.5 \sigma$ or $>2 \sigma$ deviations in a few instances where strong flaring was present, and confirmed through manual inspection. Such periods were then excluded through application of the deflare-generated good-time intervals as a filter to the level 2 event file.

An updated point-source list was then compiled through running wavdetect on a clean $0.3-7 \mathrm{keV}$ image with scales of $1,1.4,2,2.8$, and 4 pixels, using a $1.5 \mathrm{keV}$ exposure map and a threshold significance of $10^{-6}$ (which corresponds to approximately one false detection expected per chip). Net count rates were calculated for each source from aperture photometry conducted within $95 \%$ encircled energy radii (at $1.5 \mathrm{keV}$ ), with local background estimated from the median of eight nearby regions. For Field galaxies lacking a nuclear X-ray detection, the $95 \%$ confidence upper limit is estimated from the local background. Where the number of background counts is less than 10 we use the Bayesian formalism of Kraft et al. (1991) to determine the limit; elsewhere, we use Equation (9) from Gehrels (1986).

As they are the brightest galaxies within our volume-limited sample, the archival sources are generally more luminous, more massive, and thus able to retain more hot gas. For those objects, it is advantageous to determine the nuclear SMBH $\mathrm{X}$-ray luminosity from the hard-band counts, so as to minimize contamination from the diffuse soft emission. The diffuse emission may be modeled as thermal (hot gas) and powerlaw (unresolved LMXBs) components (e.g., G10; Boroson et al. 2011). For these galaxies, the contribution from hot gas generally drops below $\sim 5 \%$ of the nuclear $\mathrm{X}$-ray flux at energies $\lesssim 2 \mathrm{keV}$. Therefore, for archival galaxies with sufficiently high signal-to-noise data, defined as $\geqslant 50$ counts in the $0.3-7 \mathrm{keV}$ band (29/42 objects), we calculate X-ray flux from the $2-7 \mathrm{keV}$ count rate. ${ }^{9}$ In a few instances for which strong diffuse emission overlapped the nucleus even in the hard band, the local background was manually adjusted.

\section{RESULTS}

\subsection{Nuclear X-Ray Census}

X-ray properties of the AMUSE-Field sample are listed in Table 2, and notes on selected archival objects are given in the Appendix. From the 103 field galaxies, a nuclear X-ray source is detected in 52 cases, for a detection fraction of $50 \%$. The detection fraction is naturally higher for the archival objects

\footnotetext{
9 There are two exceptions: one snapshot object, ESO 540-014, has sufficien counts to permit use of the 2-7 keV rate, and one archival object, NGC 1052, has a sufficiently hard observed spectrum that we use the $0.3-7 \mathrm{keV}$ rate to avoid overestimating the X-ray flux.
} 
Table 1

Optical Properties

\begin{tabular}{|c|c|c|c|c|c|c|c|c|c|c|c|}
\hline Name & Type & $\begin{array}{l}\text { R.A. } \\
\text { (deg) }\end{array}$ & $\begin{array}{l}\text { Decl. } \\
\text { (deg) }\end{array}$ & $\begin{array}{c}d \\
(\mathrm{Mpc})\end{array}$ & $\begin{array}{c}B_{\mathrm{T}} \\
(\mathrm{mag})\end{array}$ & $\begin{array}{c}M_{\mathrm{B}} \\
(\mathrm{mag})\end{array}$ & $\begin{array}{c}\sigma \\
\left(\mathrm{km} \mathrm{s}^{-1}\right)\end{array}$ & $\begin{array}{l}M_{\text {star }} \\
\left(M_{\odot}\right)\end{array}$ & $\begin{array}{l}M_{\mathrm{BH}} \\
\left(M_{\odot}\right)\end{array}$ & $n_{\text {group }}$ & $\begin{array}{c}\sigma_{\text {group }} \\
\left(\mathrm{km} \mathrm{s}^{-1}\right)\end{array}$ \\
\hline NGC 1407 & $\mathrm{E}$ & 55.049583 & -18.580278 & 23.2 & 10.4 & -21.5 & 272 & 11.5 & 8.7 & 25 & 167 \\
\hline NGC 5322 & E & 207.313750 & 60.190556 & 29.4 & 11.0 & -21.4 & 233 & 11.4 & 8.5 & 21 & 169 \\
\hline NGC 4125 & $\mathrm{E}$ & 182.025000 & 65.174167 & 22.9 & 10.5 & -21.3 & 227 & 11.4 & 8.4 & 16 & 85 \\
\hline NGC 5846 & $\mathrm{E}$ & 226.622083 & 1.605556 & 26.2 & 10.8 & -21.3 & 239 & 11.4 & 8.5 & 74 & 229 \\
\hline NGC 2768 & $\mathrm{E}$ & 137.906250 & 60.037222 & 23.1 & 10.6 & -21.2 & 181 & 11.5 & 8.1 & 10 & 126 \\
\hline NGC 3923 & E & 177.757083 & -28.806111 & 20.4 & 10.4 & -21.1 & 257 & 11.4 & 8.6 & 26 & 159 \\
\hline NGC 4697 & $\mathrm{E}$ & 192.149583 & -5.800833 & 17.8 & 10.1 & -21.1 & 171 & 11.4 & 8.0 & 37 & 109 \\
\hline NGC 4494 & $\mathrm{E}$ & 187.850417 & 25.775278 & 20.9 & 10.6 & -21.0 & 150 & 11.4 & 7.7 & 11 & 83 \\
\hline IC 1459 & $\mathrm{E}$ & 344.294167 & -36.462222 & 23.2 & 10.9 & -21.0 & 306 & 11.4 & 8.9 & 16 & 221 \\
\hline NGC 5077 & $\mathrm{E}$ & 199.882083 & -12.656944 & 40.2 & 12.1 & -21.0 & 256 & 11.3 & 8.6 & 9 & 111 \\
\hline NGC 0821 & $\mathrm{E}$ & 32.087917 & 10.995000 & 25.0 & 11.2 & -20.7 & 200 & 11.1 & 8.2 & 2 & $\ldots$ \\
\hline NGC 0720 & $\mathrm{E}$ & 28.252083 & -13.738611 & 22.8 & 11.1 & -20.7 & 241 & 11.2 & 8.5 & 6 & 145 \\
\hline NGC 3585 & $\mathrm{E}$ & 168.321250 & -26.754722 & 17.8 & 10.5 & -20.7 & 206 & 11.1 & 8.3 & 9 & 70 \\
\hline NGC 3610 & $\mathrm{E}$ & 169.605417 & 58.786389 & 27.8 & 11.6 & -20.7 & 162 & 11.0 & 7.9 & 19 & 119 \\
\hline NGC 3379 & $\mathrm{E}$ & 161.956667 & 12.581667 & 13.6 & 10.1 & -20.5 & 209 & 11.1 & 8.3 & 27 & 233 \\
\hline NGC 4636 & $\mathrm{E}$ & 190.707500 & 2.687778 & 14.5 & 10.3 & -20.5 & 203 & 11.1 & 8.2 & 32 & 73 \\
\hline NGC 7507 & $\mathrm{E}$ & 348.031667 & -28.539722 & 21.0 & 11.1 & -20.5 & 222 & 11.1 & 8.4 & 4 & 80 \\
\hline NGC 1332 & E-SO & 51.572083 & -21.335278 & 20.0 & 11.0 & -20.5 & 321 & 11.1 & 9.0 & 22 & 183 \\
\hline NGC 4036 & E-SO & 180.361667 & 61.895833 & 23.2 & 11.4 & -20.4 & 181 & 11.1 & 8.1 & 16 & 92 \\
\hline NGC 3640 & E & 170.278750 & 3.234722 & 19.0 & 11.1 & -20.3 & 181 & 10.9 & 8.1 & 12 & 174 \\
\hline NGC 1052 & $\mathrm{E}$ & 40.270000 & -8.255833 & 19.7 & 11.3 & -20.1 & 207 & 11.1 & 8.3 & 22 & 103 \\
\hline NGC 5576 & $\mathrm{E}$ & 215.265417 & 3.271111 & 22.7 & 11.6 & -20.1 & 171 & 10.8 & 7.9 & 12 & 116 \\
\hline NGC 4291 & E & 185.075833 & 75.370833 & 28.7 & 12.2 & -20.1 & 285 & 10.9 & 8.8 & 8 & 92 \\
\hline NGC 5838 & E-SO & 226.359583 & 2.099444 & 20.7 & 11.5 & -20.0 & 266 & 11.0 & 8.7 & 9 & 54 \\
\hline NGC 5638 & $\mathrm{E}$ & 217.418333 & 3.233333 & 24.8 & 12.0 & -20.0 & 165 & 10.8 & 7.9 & 12 & 84 \\
\hline NGC 5831 & $\mathrm{E}$ & 226.029167 & 1.220000 & 24.9 & 12.1 & -19.8 & 165 & 10.7 & 7.9 & 74 & 229 \\
\hline NGC 3384 & E-SO & 162.070417 & 12.629167 & 13.1 & 10.8 & -19.8 & 148 & 10.8 & 7.7 & 27 & 233 \\
\hline NGC 3115 & E-SO & 151.308333 & -7.718611 & 8.4 & 9.9 & -19.8 & 268 & 10.8 & 8.7 & 5 & 58 \\
\hline NGC 3193 & E & 154.603750 & 21.893889 & 20.8 & 11.9 & -19.7 & 194 & 10.7 & 8.2 & 13 & 112 \\
\hline NGC 4203 & E-SO & 183.771250 & 33.197222 & 18.1 & 11.7 & -19.6 & 162 & 11.1 & 7.9 & 2 & $\ldots$ \\
\hline NGC 1439 & E & 56.208333 & -21.920556 & 21.3 & 12.1 & -19.5 & 150 & 10.6 & 7.7 & 24 & 121 \\
\hline NGC 5582 & $\mathrm{E}$ & 215.179583 & 39.693611 & 23.9 & 12.4 & -19.5 & 137 & 10.6 & 7.6 & 2 & $\ldots$ \\
\hline NGC 1340 & $\mathrm{E}$ & 52.082083 & -31.068056 & 12.8 & 11.1 & -19.4 & 166 & 10.6 & 7.9 & 3 & $\ldots$ \\
\hline NGC 4278 & $\mathrm{E}$ & 185.028333 & 29.280833 & 11.3 & 10.9 & -19.4 & 237 & 10.6 & 8.5 & 28 & 90 \\
\hline NGC 4742 & $\mathrm{E}$ & 192.950000 & -10.454722 & 17.9 & 11.9 & -19.4 & 108 & 10.4 & 7.2 & 37 & 109 \\
\hline NGC 2778 & $\mathrm{E}$ & 138.101667 & 35.027500 & 30.9 & 13.2 & -19.2 & 162 & 10.5 & 7.9 & 3 & $\ldots$ \\
\hline NGC 4648 & $\mathrm{E}$ & 190.435000 & 74.420833 & 25.7 & 12.8 & -19.2 & 221 & 10.5 & 8.4 & 8 & 52 \\
\hline NGC 3377 & $\mathrm{E}$ & 161.926250 & 13.985833 & 10.6 & 11.0 & -19.2 & 139 & 10.5 & 7.6 & 27 & 233 \\
\hline NGC 1426 & $\mathrm{E}$ & 55.704583 & -22.108333 & 18.0 & 12.2 & -19.1 & 151 & 10.4 & 7.7 & 24 & 121 \\
\hline NGC 1172 & $\mathrm{E}$ & 45.400000 & -14.836667 & 19.9 & 12.4 & -19.0 & 112 & 10.2 & 7.2 & 2 & $\ldots$ \\
\hline NGC 6017 & $\mathrm{E}$ & 239.314167 & 5.998333 & 27.3 & 13.5 & -18.6 & 113 & 10.3 & 7.3 & 2 & $\ldots$ \\
\hline NGC 5845 & $\mathrm{E}$ & 226.503333 & 1.633889 & 21.9 & 13.2 & -18.5 & 238 & 10.3 & 8.5 & 74 & 229 \\
\hline NGC 3457 & $\mathrm{E}$ & 163.702500 & 17.621111 & 17.7 & 12.8 & -18.4 & 71 & 10.1 & 6.5 & 31 & 124 \\
\hline ESO 576-076 & $\mathrm{E}$ & 202.678750 & -22.421111 & 23.0 & 13.5 & -18.3 & 98 & 10.0 & 7.0 & 12 & 141 \\
\hline NGC 4283 & $\mathrm{E}$ & 185.086667 & 29.310833 & 16.4 & 12.9 & -18.2 & 114 & 10.1 & 7.3 & 14 & 102 \\
\hline NGC 3928 & $\mathrm{E}$ & 177.948333 & 48.683056 & 17.2 & 13.1 & -18.1 & 113 & 9.9 & 7.3 & 21 & 65 \\
\hline NGC 3641 & $\mathrm{E}$ & 170.286667 & 3.194722 & 25.5 & 13.9 & -18.1 & 163 & 10.0 & 7.9 & 12 & 174 \\
\hline NGC 4121 & $\mathrm{E}$ & 181.985833 & 65.113889 & 24.0 & 13.8 & -18.1 & 84 & 10.0 & 6.8 & 16 & 85 \\
\hline UGC07767 & $\mathrm{E}$ & 188.885000 & 73.674722 & 23.2 & 13.8 & -18.1 & 131 & 10.0 & 7.5 & 8 & 52 \\
\hline NGC 3265 & $\mathrm{E}$ & 157.778333 & 28.796667 & 22.4 & 13.9 & -17.8 & $\ldots$ & 9.8 & 7.1 & 6 & 39 \\
\hline IC 1729 & $\mathrm{E}$ & 26.980417 & -26.892222 & 17.5 & 13.4 & -17.8 & 133 & 10.0 & 7.5 & 1 & $\ldots$ \\
\hline NGC 2970 & $\mathrm{E}$ & 145.879583 & 31.976944 & 25.2 & 14.3 & -17.7 & 43 & 9.6 & 5.6 & 9 & 91 \\
\hline UGC 05955 & E & 163.017917 & 71.773056 & 21.7 & 14.0 & -17.6 & 83 & 9.7 & 6.8 & 1 & $\ldots$ \\
\hline NGC 3073 & E-SO & 150.217083 & 55.618889 & 20.3 & 14.0 & -17.5 & 35 & 9.4 & 5.3 & 7 & 94 \\
\hline NGC 3522 & E & 166.668750 & 20.085556 & 18.8 & 14.0 & -17.4 & 87 & 9.6 & 6.8 & 1 & $\ldots$ \\
\hline NGC 1097A & $\mathrm{E}$ & 41.541250 & -30.228056 & 16.2 & 13.9 & -17.2 & $\ldots$ & 9.5 & 6.8 & 4 & 140 \\
\hline NGC 4627 & $\mathrm{E}$ & 190.498750 & 32.573611 & 10.4 & 12.9 & -17.1 & 44 & 9.6 & 5.7 & 28 & 90 \\
\hline NGC 1370 & $\mathrm{E}$ & 53.810833 & -20.373611 & 12.6 & 13.5 & -17.0 & 71 & 9.6 & 6.5 & 1 & $\ldots$ \\
\hline NGC 0855 & $\mathrm{E}$ & 33.514583 & 27.877222 & 9.7 & 13.0 & -17.0 & 63 & 9.2 & 6.3 & 1 & $\ldots$ \\
\hline NGC 7077 & $\mathrm{E}$ & 322.498333 & 2.414167 & 17.1 & 14.2 & -16.9 & 41 & 9.1 & 5.6 & 1 & $\ldots$ \\
\hline PGC 056821 & $\mathrm{E}$ & 240.697917 & 19.787222 & 26.2 & 15.2 & -16.9 & $\ldots$ & 9.6 & 6.7 & $\ldots$ & $\ldots$ \\
\hline IC 0225 & $\mathrm{E}$ & 36.617917 & 1.160556 & 21.1 & 14.7 & -16.9 & $\ldots$ & 9.1 & 6.7 & 12 & 129 \\
\hline NGC 1331 & $\mathrm{E}$ & 51.617917 & -21.355278 & 14.8 & 14.1 & -16.7 & 57 & 9.4 & 6.1 & 22 & 183 \\
\hline ESO 540-014 & $\mathrm{E}$ & 10.298750 & -21.131667 & 21.8 & 15.0 & -16.6 & $\ldots$ & 8.6 & 6.6 & 1 & $\ldots$ \\
\hline PGC 042748 & $\mathrm{E}$ & 190.734583 & 3.676667 & 14.6 & 14.5 & -16.3 & 39 & 9.2 & 5.5 & 32 & 73 \\
\hline
\end{tabular}


Table 1

(Continued)

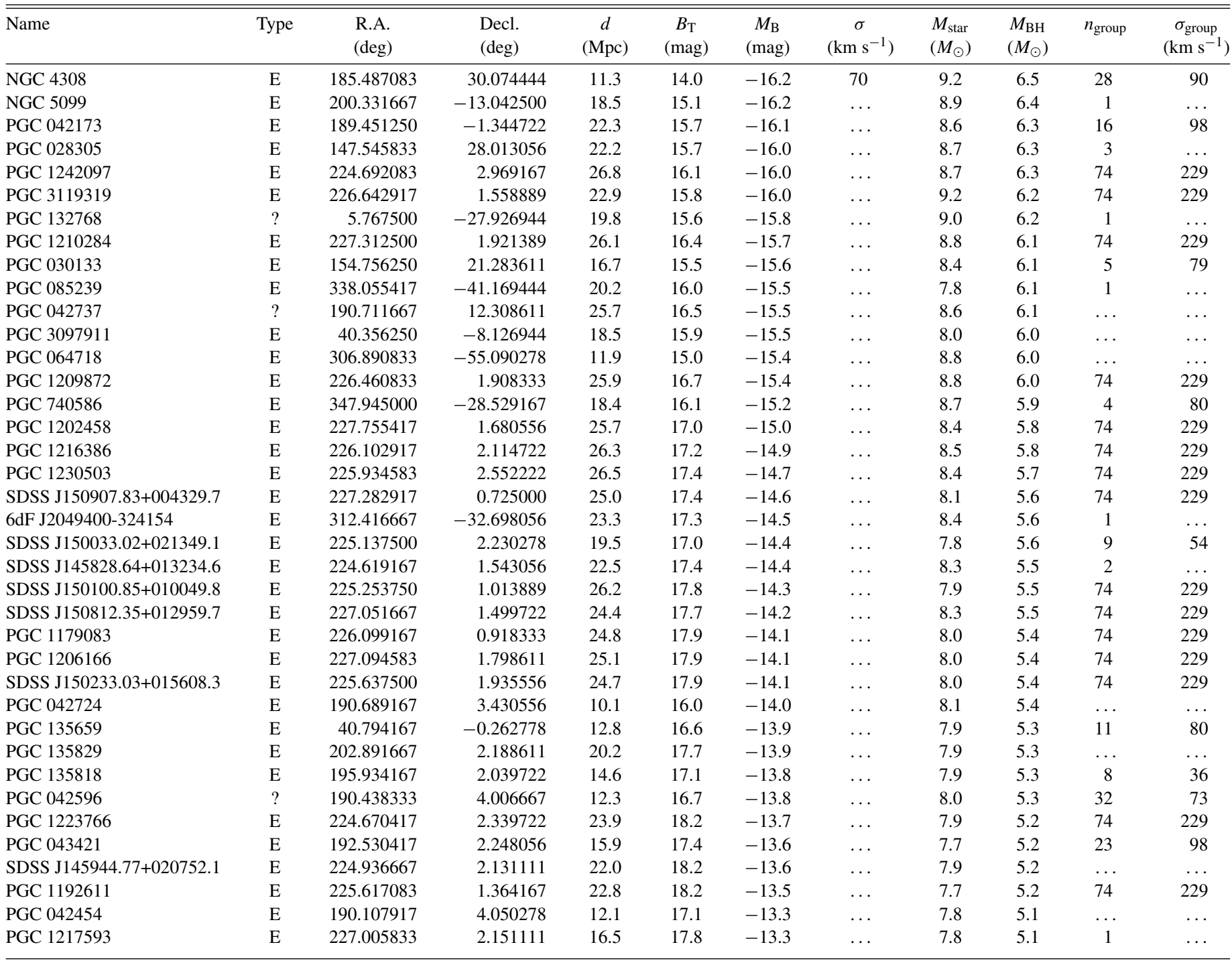

Notes. The columns Name, Type, R.A., Decl., $d, B_{\mathrm{T}}, M_{\mathrm{B}}$, and $\sigma$ are taken directly or indirectly from the HyperLeda database. Stellar and black hole masses $M_{\text {star }}$ and $M_{\mathrm{BH}}$ are given here as logarithm quantities and calculated as described in Section 4.1. Group size and velocity dispersions are from Makarov \& Karachentsev (2011). Rows are ordered by absolute $B$ magnitude.

(33/42 or $79 \%)$, owing to their generally deeper exposures. ${ }^{10}$ References for previously published analysis of the archival objects are also listed in Table 2.

The detection fraction for the snapshot objects is $19 / 61$ or $31 \%$. Cutout images of all snapshot targets with detected nuclear $\mathrm{X}$-ray emission are shown in Figure 1, smoothed with a Gaussian kernel of five pixels. Count rates (either $0.3-7 \mathrm{keV}$ or 2-7 keV, as discussed in Section 2) were converted to $0.3-10 \mathrm{keV}$ fluxes with PIMMS, ${ }^{11}$ assuming a power-law spectrum with $\Gamma=2$ and Galactic absorption. For each galaxy the radial-velocity distance modulus in HyperLeda was used to calculate the nuclear $0.3-10 \mathrm{keV}$ X-ray luminosity, $L_{\mathrm{X}}$. For most of these objects, non-redshift distances are not available in HyperLeda or NED; from the remainder, the non-redshift distance modulus

\footnotetext{
${ }^{10}$ Of the nine archival upper limits, five have effective exposure times less than $10 \mathrm{ks}$, two are small galaxies serendipitously observed off-axis $\left(\theta \gtrsim 2^{\prime} .5\right)$, and two (NGC 5846 and NGC 3923; see the Appendix) do not show a cleanly resolved central point source.

11 http://cxc.harvard.edu/toolkit/pimms.jsp
}

from HyperLeda is an average of 0.16 lower, corresponding to a distance $\sim 1.5 \mathrm{Mpc}$ closer at $d \sim 20 \mathrm{Mpc}$. For the low redshift of these objects, the $k$-correction is insignificant and is therefore neglected.

Nine field galaxies have $\log L_{\mathrm{X}} \gtrsim 39.8$ : NGC 1052, 2768, 4036, 4203, 4278, 4494, 5077, IC 1459, and ESO 540-014. All but the last of these are from the archival sample. Each one of these nine galaxies has radio and/or optical properties consistent with the presence of low-level AGN activity. NGC 1052, 4036, 4278, 4494, and IC 1459 are included as LINER/AGNs in González-Martín et al. (2009); NGC 1052, 4278, 5077, and IC 1459 are categorized as flat-spectrum radio sources in Healey et al. (2007); NGC 2768, 4036, 4203, and ESO 540-014 are identified as active in Véron-Cetty \& Véron (2006) as Seyfert 1, LINER b, LINER b, and Seyfert 2 galaxies, respectively. In comparison, the AMUSE-Virgo sample contains only two objects (M87 and NGC 4564) with $\log L_{\mathrm{X}}>39.8$ (from Table 1 of G10). 
Table 2

X-Ray Properties

\begin{tabular}{|c|c|c|c|c|c|c|c|c|}
\hline Name & ObsID & $\begin{array}{l}\text { Band } \\
(\mathrm{keV})\end{array}$ & $\begin{array}{c}\theta \\
\left({ }^{\prime}\right)\end{array}$ & $\begin{array}{l}\text { Exp } \\
(\mathrm{ks})\end{array}$ & Counts & $\begin{array}{l}\text { Rate } \\
\left(\mathrm{ks}^{-1}\right)\end{array}$ & $\begin{array}{c}\log L_{\mathrm{X}} \\
\left(\mathrm{erg} \mathrm{s}^{-1}\right)\end{array}$ & References $^{\mathrm{a}}$ \\
\hline NGC 1407 & 791 & $2-7$ & 0.0 & 35.4 & 44.1 & 1.2 & 39.5 & 1 \\
\hline NGC 5322 & 6787 & $2-7$ & 0.0 & 13.8 & 9.0 & 0.7 & 39.5 & 2 \\
\hline NGC 4125 & 2071 & $2-7$ & 0.1 & 61.6 & 21.9 & 0.4 & 39.0 & 3 \\
\hline NGC 5846 & 788 & $2-7$ & 0.1 & 23.1 & 5.4 & $<0.3$ & $<39.0$ & 4 \\
\hline NGC 2768 & 9528 & $2-7$ & 0.0 & 64.1 & 140.3 & 2.4 & 39.8 & 3 \\
\hline NGC 3923 & 9507 & $2-7$ & 0.1 & 80.6 & 23.0 & $<0.1$ & $<38.5$ & 5 \\
\hline NGC 4697 & 4730 & $2-7$ & 0.0 & 38.2 & 26.3 & 0.8 & 39.1 & 6 \\
\hline NGC 4494 & 2079 & $2-7$ & 0.1 & 15.0 & 61.7 & 4.6 & 40.0 & 7 \\
\hline IC 1459 & 2196 & $2-7$ & 0.0 & 52.7 & 1706.5 & 36.0 & 41.0 & 8 \\
\hline NGC 5077 & 11780 & $2-7$ & 0.0 & 28.8 & 31.7 & 1.2 & 40.0 & 9 \\
\hline NGC 0821 & 6313 & $2-7$ & 0.0 & 49.3 & 11.7 & 0.3 & 39.0 & 3 \\
\hline NGC 0720 & 7372 & $2-7$ & 0.0 & 47.7 & 29.3 & 0.7 & 39.3 & 10 \\
\hline NGC 3585 & 9506 & $2-7$ & 0.1 & 59.2 & 30.7 & 0.6 & 38.9 & 8 \\
\hline NGC 3610 & 7141 & $0.3-7$ & 0.0 & 4.9 & 15.1 & 3.2 & 39.3 & 11 \\
\hline NGC 3379 & 7073 & $2-7$ & 0.0 & 81.3 & 37.2 & 0.5 & 38.6 & 12 \\
\hline NGC 4636 & 323 & $2-7$ & 0.0 & 43.5 & 13.5 & 0.2 & 38.3 & 13 \\
\hline NGC 7507 & 11344 & $0.3-7$ & 0.1 & 7.3 & 18.7 & 2.7 & 39.0 & 14 \\
\hline NGC 1332 & 4372 & $2-7$ & 0.1 & 31.7 & 28.4 & 0.8 & 39.2 & 15 \\
\hline NGC 4036 & 6783 & $2-7$ & 0.0 & 13.7 & 83.2 & 6.7 & 40.3 & 11 \\
\hline NGC 3640 & 7142 & $0.3-7$ & 0.0 & 9.0 & 7.3 & $<0.7$ & $<38.3$ & 11 \\
\hline NGC 1052 & 5910 & $0.3-7$ & 0.0 & 55.3 & 4952.3 & 94.2 & 40.6 & 3 \\
\hline NGC 5576 & 11781 & $0.3-7$ & 0.0 & 29.7 & 41.9 & 1.5 & 38.8 & 9 \\
\hline NGC 4291 & 11778 & $2-7$ & 0.0 & 29.6 & 16.8 & 0.6 & 39.4 & 9 \\
\hline NGC 5838 & 6788 & $2-7$ & 0.0 & 13.6 & 15.9 & 1.3 & 39.5 & 11 \\
\hline NGC 5638 & 11313 & $0.3-7$ & 0.0 & 10.0 & 3.0 & $<0.4$ & $<38.3$ & 14 \\
\hline NGC 5831 & 11314 & $0.3-7$ & 3.5 & 9.6 & 36.7 & 3.8 & 39.4 & 14 \\
\hline NGC 3384 & 11782 & $2-7$ & 0.0 & 28.6 & 20.9 & 0.8 & 38.8 & 9 \\
\hline NGC 3115 & 12095 & $2-7$ & 0.0 & 76.0 & 80.1 & 1.2 & 38.6 & 16 \\
\hline NGC 3193 & 11360 & $0.3-7$ & 0.0 & 7.2 & 18.6 & 2.7 & 39.0 & 14 \\
\hline NGC 4203 & 10535 & $2-7$ & 0.0 & 41.4 & 2022.2 & 54.2 & 40.9 & 17 \\
\hline NGC 1439 & 11346 & $0.3-7$ & 0.0 & 7.5 & 19.0 & 2.6 & 39.1 & 14 \\
\hline NGC 5582 & 11361 & $0.3-7$ & 0.0 & 9.5 & 9.3 & 1.0 & 38.7 & 14 \\
\hline NGC 1340 & 11345 & $0.3-7$ & 0.0 & 2.9 & 2.1 & $<1.1$ & $<38.2$ & 14 \\
\hline NGC 4278 & 7081 & $2-7$ & 0.0 & 110.1 & 966.4 & 9.7 & 39.8 & 18 \\
\hline NGC 4742 & 11779 & $2-7$ & 0.0 & 32.8 & 37.0 & 1.2 & 39.3 & 9 \\
\hline NGC 2778 & 11777 & $0.3-7$ & 0.0 & 29.3 & 28.9 & 1.0 & 38.9 & 9 \\
\hline NGC 4648 & 11362 & $0.3-7$ & 0.0 & 10.8 & 15.5 & 1.5 & 39.0 & 14 \\
\hline NGC 3377 & 2934 & $2-7$ & 0.2 & 37.6 & 24.0 & 0.7 & 38.6 & 19 \\
\hline NGC 1426 & 11347 & $0.3-7$ & 0.0 & 5.4 & 2.8 & $<0.6$ & $<38.3$ & 14 \\
\hline NGC 1172 & 11348 & $0.3-7$ & 0.0 & 6.5 & 4.0 & 0.6 & 38.4 & 14 \\
\hline NGC 6017 & 11363 & $0.3-7$ & 0.1 & 12.0 & 29.5 & 2.6 & 39.3 & 14 \\
\hline NGC 5845 & 4009 & $2-7$ & 0.0 & 29.8 & 23.9 & 0.9 & 39.4 & 20 \\
\hline NGC 3457 & 11364 & $0.3-7$ & 0.1 & 5.2 & 7.8 & 1.5 & 38.7 & 14 \\
\hline ESO 576-076 & 11316 & $0.3-7$ & 0.0 & 8.8 & 1.0 & $<0.4$ & $<38.4$ & 14 \\
\hline NGC 4283 & 7081 & $2-7$ & 3.5 & 106.7 & 52.7 & 0.5 & 38.9 & 18 \\
\hline NGC 3928 & 11365 & $0.3-7$ & 0.0 & 4.5 & 5.7 & 1.3 & 38.6 & 14 \\
\hline NGC 3641 & 7142 & $0.3-7$ & 2.5 & 8.8 & 8.0 & 1.0 & 38.8 & \\
\hline NGC 4121 & 2071 & $0.3-7$ & 3.8 & 51.6 & 17.0 & 0.3 & 38.0 & \\
\hline UGC 07767 & 11367 & $0.3-7$ & 0.0 & 8.5 & 5.6 & 0.7 & 38.5 & 14 \\
\hline NGC 3265 & 11368 & $0.3-7$ & 0.0 & 8.3 & 17.2 & 2.2 & 39.0 & 14 \\
\hline IC 1729 & 11349 & $0.3-7$ & 0.0 & 5.5 & 17.0 & 3.2 & 39.0 & 14 \\
\hline NGC 2970 & 11369 & $0.3-7$ & 0.0 & 10.5 & 8.0 & 0.8 & 38.7 & 14 \\
\hline UGC 05955 & 11370 & $0.3-7$ & 0.0 & 7.4 & 3.4 & $<0.5$ & $<38.3$ & 14 \\
\hline NGC 3073 & 7851 & $0.3-7$ & 0.0 & 4.7 & 1.0 & $<0.8$ & $<38.4$ & \\
\hline NGC 3522 & 11371 & $0.3-7$ & 0.0 & 5.9 & 5.8 & 1.0 & 38.5 & 14 \\
\hline NGC 1097A & 1611 & $0.3-7$ & 3.4 & 5.2 & 1.0 & $<0.7$ & $<38.0$ & 21 \\
\hline NGC 4627 & 797 & $0.3-7$ & 2.5 & 56.4 & 5.0 & $<0.1$ & $<36.9$ & \\
\hline NGC 1370 & 11350 & $0.3-7$ & 0.0 & 2.7 & 8.0 & 3.1 & 38.7 & 14 \\
\hline NGC 0855 & 9550 & $2-7$ & 0.0 & 58.6 & 46.9 & 0.9 & 38.6 & \\
\hline NGC 7077 & 7854 & $0.3-7$ & 0.0 & 5.1 & 0.0 & $<0.7$ & $<38.3$ & \\
\hline PGC 056821 & 11373 & $0.3-7$ & 0.0 & 11.5 & 6.7 & 0.6 & 38.6 & 14 \\
\hline IC 0225 & 11351 & $0.3-7$ & 0.0 & 7.5 & 2.4 & $<0.5$ & $<38.3$ & 14 \\
\hline NGC 1331 & 4372 & $0.3-7$ & 2.9 & 33.2 & 16.5 & 0.5 & 37.9 & 15 \\
\hline ESO 540-014 & 11352 & $2-7$ & 0.0 & 7.9 & 33.1 & 4.7 & 40.1 & 14 \\
\hline PGC 042748 & 11318 & $0.3-7$ & 0.0 & 3.5 & 0.0 & $<1.0$ & $<38.3$ & 14 \\
\hline
\end{tabular}


Table 2

(Continued)

\begin{tabular}{|c|c|c|c|c|c|c|c|c|}
\hline Name & ObsID & $\begin{array}{l}\text { Band } \\
(\mathrm{keV})\end{array}$ & $\begin{array}{c}\theta \\
\left(^{\prime}\right)\end{array}$ & $\begin{array}{l}\text { Exp } \\
(\mathrm{ks})\end{array}$ & Counts & $\begin{array}{c}\text { Rate } \\
\left(\mathrm{ks}^{-1}\right)\end{array}$ & $\begin{array}{c}\log L_{X} \\
\left(\operatorname{erg~s}^{-1}\right)\end{array}$ & References $^{\mathrm{a}}$ \\
\hline NGC 4308 & 7853 & $0.3-7$ & 0.0 & 4.7 & 2.9 & $<0.8$ & $<37.9$ & \\
\hline NGC 5099 & 11319 & $0.3-7$ & 0.0 & 5.8 & 0.0 & $<0.6$ & $<38.3$ & 14 \\
\hline PGC 042173 & 11320 & $0.3-7$ & 0.0 & 7.9 & 0.0 & $<0.4$ & $<38.3$ & 14 \\
\hline PGC 028305 & 11376 & $0.3-7$ & 0.0 & 8.2 & 0.0 & $<0.4$ & $<38.3$ & 14 \\
\hline PGC 1242097 & 11375 & $0.3-7$ & 0.0 & 11.6 & 0.0 & $<0.3$ & $<38.3$ & 14 \\
\hline PGC 3119319 & 788 & $0.3-7$ & 3.1 & 22.2 & 5.0 & $<0.3$ & $<38.1$ & \\
\hline PGC 132768 & 11354 & $0.3-7$ & 0.0 & 6.6 & 1.0 & $<0.5$ & $<38.3$ & 14 \\
\hline PGC 1210284 & 11377 & $0.3-7$ & 0.0 & 11.2 & 1.0 & $<0.3$ & $<38.3$ & 14 \\
\hline PGC 030133 & 11378 & $0.3-7$ & 0.0 & 4.7 & 0.0 & $<0.7$ & $<38.3$ & 14 \\
\hline PGC 085239 & 11341 & $0.3-7$ & 0.0 & 6.9 & 0.0 & $<0.5$ & $<38.3$ & 14 \\
\hline PGC 042737 & 11322 & $0.3-7$ & 0.0 & 10.7 & 0.0 & $<0.3$ & $<38.3$ & 14 \\
\hline PGC 3097911 & 11355 & $0.3-7$ & 0.0 & 5.7 & 0.0 & $<0.6$ & $<38.3$ & 14 \\
\hline PGC 064718 & 11342 & $0.3-7$ & 0.0 & 2.0 & 1.0 & $<1.6$ & $<38.4$ & 14 \\
\hline PGC 1209872 & 11379 & $0.3-7$ & 0.0 & 11.1 & 1.4 & $<0.3$ & $<38.3$ & 14 \\
\hline PGC 740586 & 11344 & $0.3-7$ & 4.5 & 7.0 & 0.8 & $<0.5$ & $<38.2$ & 14 \\
\hline PGC 1202458 & 11380 & $0.3-7$ & 0.0 & 10.9 & 0.0 & $<0.3$ & $<38.3$ & 14 \\
\hline PGC 1216386 & 11381 & $0.3-7$ & 0.0 & 11.4 & 0.0 & $<0.3$ & $<38.3$ & 14 \\
\hline PGC 1230503 & 11382 & $0.3-7$ & 0.0 & 11.8 & 0.0 & $<0.3$ & $<38.3$ & 14 \\
\hline SDSS J150907.83+004329.7 & 11383 & $0.3-7$ & 0.0 & 9.4 & 0.0 & $<0.4$ & $<38.4$ & 14 \\
\hline $6 \mathrm{dF}$ J2049400-324154 & 11357 & $0.3-7$ & 0.0 & 9.0 & 0.0 & $<0.4$ & $<38.3$ & 14 \\
\hline SDSS J150033.02+021349.1 & 11324 & $0.3-7$ & 0.0 & 6.3 & 0.0 & $<0.5$ & $<38.3$ & 14 \\
\hline SDSS J145828.64+013234.6 & 11325 & $0.3-7$ & 0.0 & 8.4 & 0.0 & $<0.4$ & $<38.3$ & 14 \\
\hline SDSS J150100.85+010049.8 & 11327 & $0.3-7$ & 0.0 & 11.4 & 0.0 & $<0.3$ & $<38.3$ & 14 \\
\hline SDSS J150812.35+012959.7 & 11384 & $0.3-7$ & 0.0 & 10.0 & 1.0 & $<0.4$ & $<38.3$ & 14 \\
\hline PGC 1179083 & 11330 & $0.3-7$ & 0.1 & 9.9 & 0.0 & $<0.4$ & $<38.3$ & 14 \\
\hline PGC 1206166 & 11385 & $0.3-7$ & 0.0 & 10.5 & 7.0 & 0.7 & 38.6 & 14 \\
\hline SDSS J150233.03+015608.3 & 11329 & $0.3-7$ & 0.0 & 10.0 & 0.0 & $<0.3$ & $<38.3$ & 14 \\
\hline PGC 042724 & 11331 & $0.3-7$ & 0.0 & 1.8 & 0.0 & $<1.8$ & $<38.2$ & 14 \\
\hline PGC 135659 & 11358 & $0.3-7$ & 0.0 & 2.7 & 0.0 & $<1.2$ & $<38.3$ & 14 \\
\hline PGC 135829 & 11333 & $0.3-7$ & 0.1 & 6.7 & 0.0 & $<0.5$ & $<38.3$ & 14 \\
\hline PGC 135818 & 11334 & $0.3-7$ & 0.0 & 3.5 & 0.0 & $<0.9$ & $<38.3$ & 14 \\
\hline PGC 042596 & 11335 & $0.3-7$ & 0.0 & 2.5 & 1.0 & $<1.3$ & $<38.3$ & 14 \\
\hline PGC 1223766 & 11337 & $0.3-7$ & 0.0 & 9.2 & 3.2 & $<0.4$ & $<38.3$ & 14 \\
\hline PGC 043421 & 11338 & $0.3-7$ & 0.0 & 3.9 & 0.0 & $<0.9$ & $<38.3$ & 14 \\
\hline SDSS J145944.77+020752.1 & 11336 & $0.3-7$ & 0.0 & 8.0 & 0.0 & $<0.4$ & $<38.3$ & 14 \\
\hline PGC 1192611 & 11339 & $0.3-7$ & 0.1 & 8.4 & 0.0 & $<0.4$ & $<38.3$ & 14 \\
\hline PGC 042454 & 11340 & $0.3-7$ & 0.0 & 2.6 & 0.0 & $<1.3$ & $<38.2$ & 14 \\
\hline PGC 1217593 & 11386 & $0.3-7$ & 0.0 & 4.6 & 0.0 & $<0.7$ & $<38.3$ & 14 \\
\hline
\end{tabular}

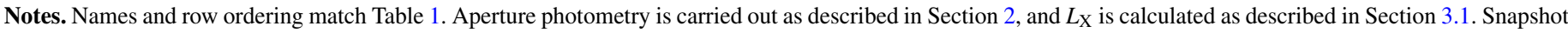
observations are those for which the reference is 14 .

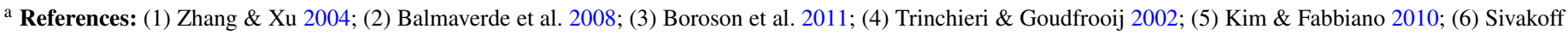

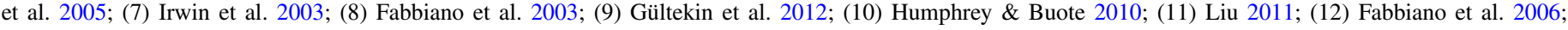

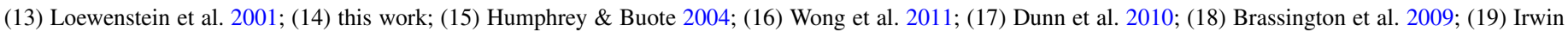
et al. 2004; (20) Soria et al. 2006a; (21) Nemmen et al. 2006.

\subsection{Origins of the Nuclear X-Ray Emission}

The correspondence between higher values of X-ray luminosity and optical or radio indicators of activity (Section 3.1) already supports that, for at least these objects, the nuclear X-ray emission is linked to the central SMBH. Indeed, prior studies of early-type galaxies (G08, G10, and references in Section 1) have overwhelmingly associated nuclear point-source $L_{\mathrm{X}}$ measurements with low-level SMBH activity. While our observations are not designed to determine the precise X-ray emission mechanism, various proposed inefficient accretion flow models (see, e.g., discussion and references in Soria et al. 2006b) may apply to the objects in the AMUSE samples. We here also consider alternative origins for the nuclear X-ray emission and find that they likely explain at most a small fraction of the observed detections.
The probability of finding an unrelated background source of equal or greater flux within the Chandra point-spread function (PSF) for any given nuclear X-ray detection is negligible, based on the $N(S)$ relation provided by Moretti et al. (2003), which Bauer et al. (2004) find matches the source count distribution within the Chandra Deep Fields. Contamination from LMXBs, while still unlikely, must be considered as a potential source of nuclear X-ray emission (Section 3.2.1). Tidal disruption of stars may provide an alternative method of fueling SMBHs (Section 3.2.2).

\subsubsection{Low-Mass X-Ray Binary Contamination}

Where a nuclear star cluster is not present, the total number of LMXBs and their cumulative X-ray luminosity scale approximately with the stellar mass (e.g., Gilfanov 2004), and so the number of LMXBs per unit stellar mass above a particular 

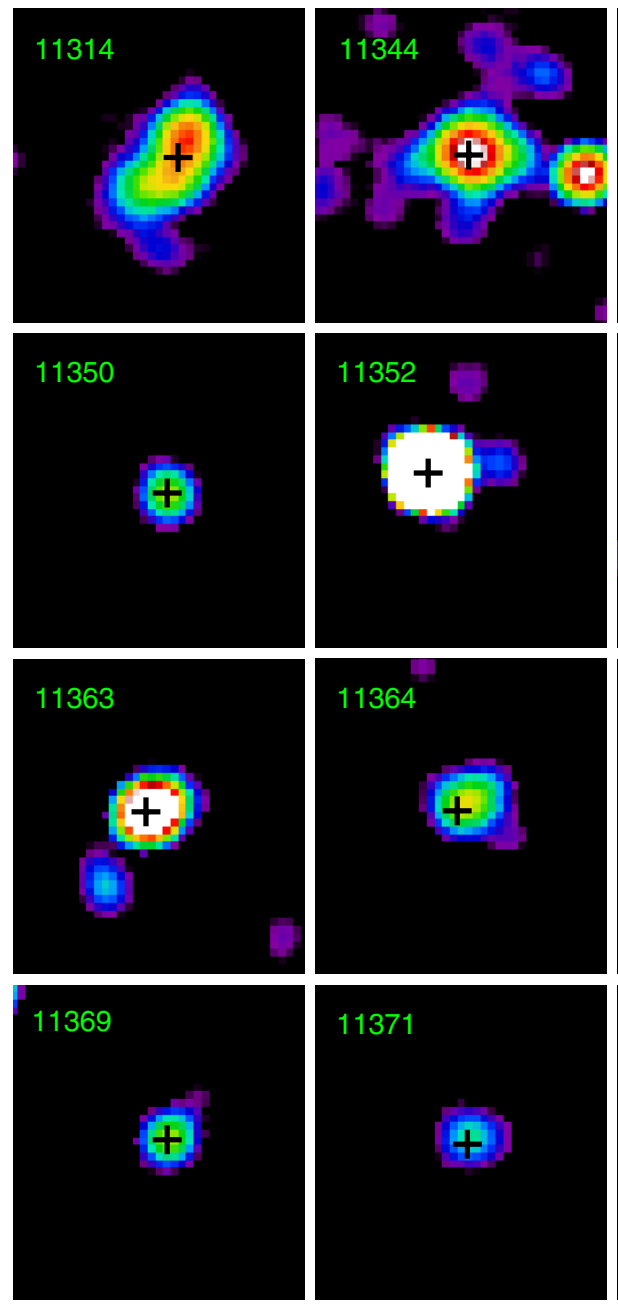
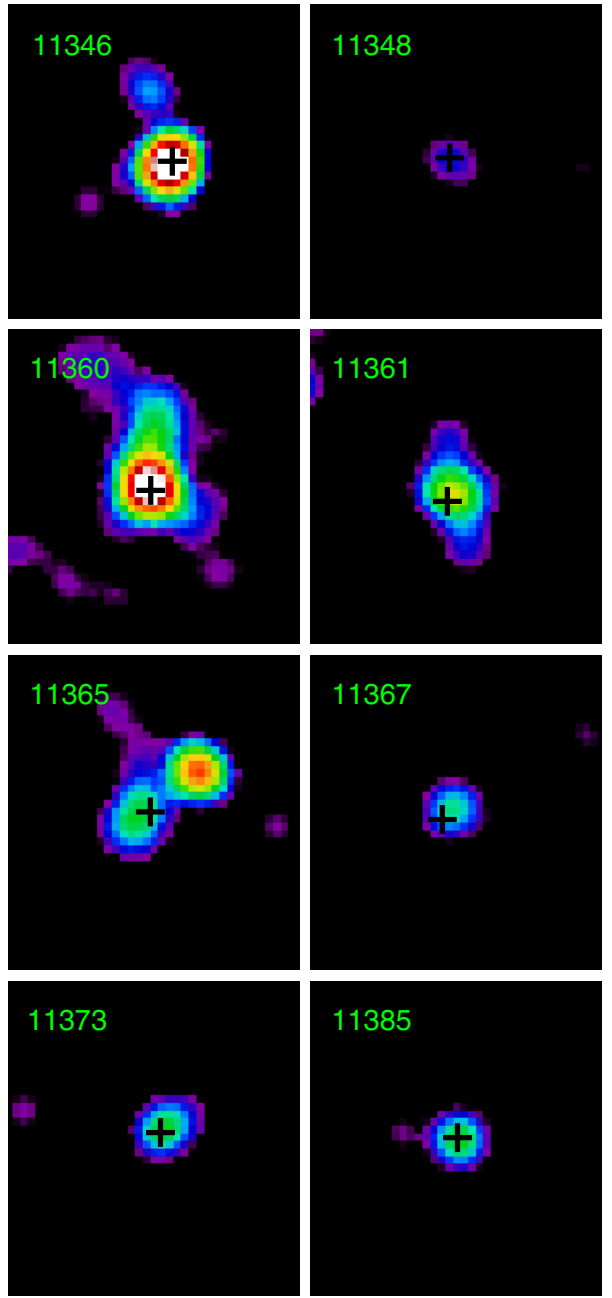
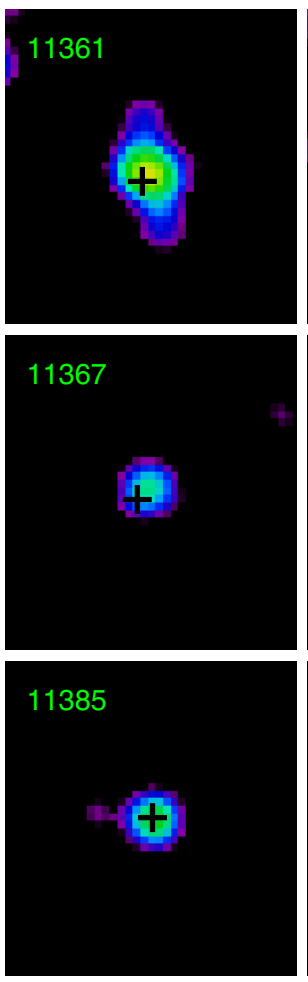
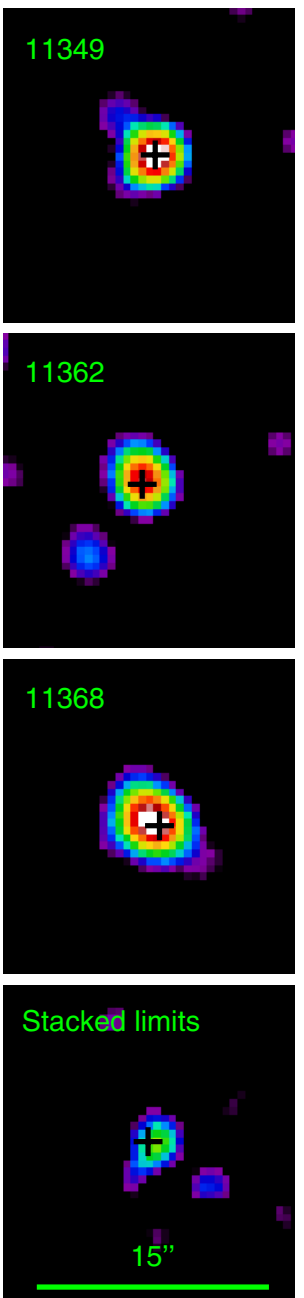

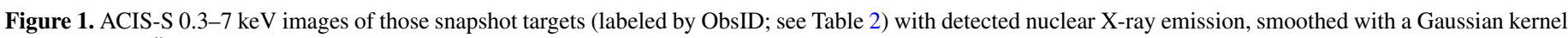

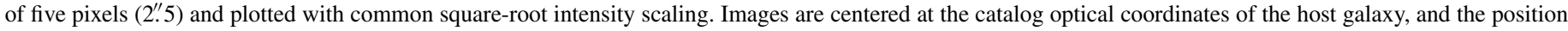

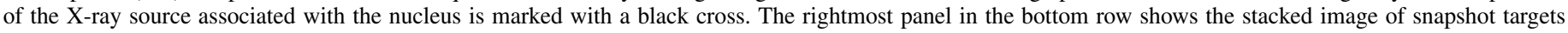

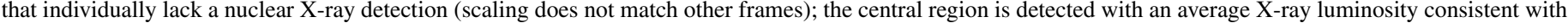
arising from a population of enclosed LMXBs.

(A color version of this figure is available in the online journal.)

luminosity threshold may be estimated from the $\mathrm{X}$-ray luminosity function for LMXBs (e.g., Gilfanov 2004). We estimate the projected stellar mass enclosed by the Chandra PSF based on the fractional luminosity within 2"; as most of the Field galaxies currently lack high-resolution $H S T$ imaging, we use as a comparative template similarly distant Virgo early-type galaxies with $-17<M_{B}<-16$ (i.e., $\log \left(M_{\text {star }} / M_{\odot}\right) \sim 9-10$ ), which tend to have effective radii $10^{\prime \prime}-20^{\prime \prime}$ with Sérsic indices of 1.0-2.5 (Ferrarese et al. 2006b). The total X-ray luminosity from LMXBs within the nucleus of a typical such galaxy is $L_{\mathrm{X}}=1.0 \times 10^{37} \mathrm{erg} \mathrm{s}^{-1}$, which is $4 \%$ of the Field detection limit. The expected nuclear contribution from LMXBs can be several times greater in large ellipticals, where the total stellar mass can be $\log \left(M_{\text {star }} / M_{\odot}\right) \sim 11$ (here the smaller fraction of the effective radius enclosed by the PSF is partially offset by a higher Sérsic index, for simple models, but see, e.g., Ferrarese et al. 2006b for discussion of surface brightness profiles), but for such galaxies in the Field sample the nuclear X-ray luminosities are generally substantially above the detection limit. In summary, owing to the steepness of the LMXB luminosity function above a few times $10^{38} \mathrm{erg} \mathrm{s}^{-1}$, for most of the Field galaxies it is highly unlikely that the PSF-enclosed stel- lar mass would generate significant LMXB contribution to the measured $L_{\mathrm{X}}$ values. Figure 2 shows the $\log L_{\mathrm{X}}$ number density for the Field sample, which may be satisfactorily modeled as $d N / d \log L_{\mathrm{X}}=100 \times\left(L_{\mathrm{X}} / 10^{38}\right)^{-0.6}$. As for the Virgo sample (see Figure 6 of G10), this is much shallower than would be expected if most of the X-ray detections were actually LMXB dominated.

Massive nuclear star clusters, with inferred radii around a few tens of pc, become increasingly prominent down the mass function (e.g., Bekki \& Graham 2010; Graham et al. 2011; and references therein). The enhanced stellar encounter rates within a nuclear star cluster imply a larger possibility of chance LMXB contamination. To account for this effect, for nucleated galaxies G10 conservatively adopted the higher $\mathrm{X}$-ray luminosity function of LMXBs in globular clusters, as estimated by Sivakoff et al. (2007). Unfortunately, most of the field galaxies discussed here currently lack the two-color highresolution optical imaging (i.e., carried out with HST ACS) necessary to confirm the presence of a nuclear star cluster and to infer its mass.

To estimate the degree of LMXB contamination within the field sample detections, we note that the AMUSE-Virgo sample 


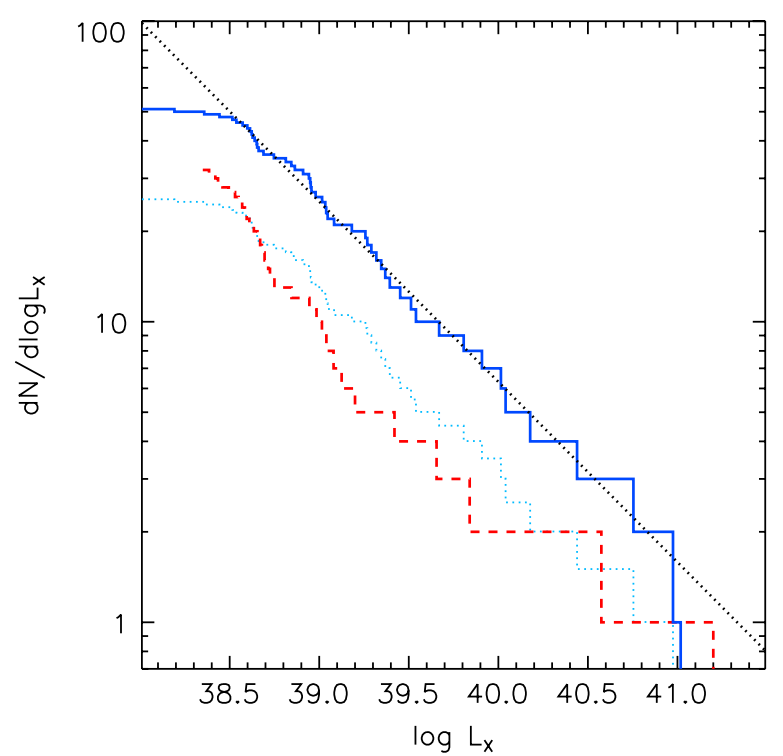

Figure 2. $\log L_{X}$ number density for the AMUSE-Field sample (solid blue line), which may be modeled as $d N / d \log L_{\mathrm{X}}=100 \times\left(L_{\mathrm{X}} / 10^{38}\right)^{-0.6}($ dotted black line). For comparison, the result for the AMUSE-Virgo sample (dashed red line) is plotted along with the Field number density rescaled by 0.5 (dotted cyan line). The luminosity function for LMXBs is much steeper (see, e.g., Figure 6 of G10), confirming that in almost all cases the X-ray detections are linked to low-level SMBH activity.

(A color version of this figure is available in the online journal.)

contained 6/32 objects with both a nuclear star cluster and an X-ray detection. All six of these objects had $\log \left(M_{\text {star }} / M_{\odot}\right)<$ 10.5 (and in total there are 16 Virgo X-ray detections with $\left.\log \left(M_{\text {star }} / M_{\odot}\right)<10.5\right)$. The influence of nuclear star clusters at higher stellar masses, even if present, is of decreasing relevance; Graham \& Spitler (2009) find that in mixed nuclei the SMBH mass dominates that of the nuclear star cluster for spheroid masses above $\sim 10^{10} M_{\odot}$. In 4/6 of the Virgo objects with both a nuclear star cluster and an X-ray detection (i.e., in 4/16 of Virgo X-ray-detected objects with $\left.\log \left(M_{\text {star }} / M_{\odot}\right)<10.5\right)$, G10 calculate that the X-ray emission was likely due to LMXB rather than SMBH activity. Applying a similar rate ${ }^{12}$ of $4 / 16=0.25$ to the 22 Field galaxies with $\log \left(M_{\text {star }} / M_{\odot}\right)<10.5$ and X-ray detections suggests that in $\lesssim 6$ cases the $\mathrm{X}$-ray emission might be contaminated by a bright LMXB; Figure 4 shows one plausible distribution of intrinsic SMBH detections in the Field sample (here the galaxies with presumed nuclear star cluster LMXB contamination all have $L_{\mathrm{X}}<5 \times 10^{38} \mathrm{erg} \mathrm{s}^{-1}$ ). The detected intrinsic fraction of accreting SMBHs within the Field sample is then $\sim 46 / 103$ (rather than the observed 52/103 rate of $\mathrm{X}$-ray-detected nuclei), or $45 \% \pm 7 \%$, which sets a lower limit to the occupation fraction of SMBHs within these galaxies.

Off-nuclear X-ray sources from many of the archival observations are discussed elsewhere (e.g., Irwin et al. 2003). Within the snapshot observations, several off-nuclear X-ray sources are detected within three times the effective radius (this limit is sometimes used to construct catalogs of ultraluminous X-ray sources (ULXs) or LMXBs, e.g., Irwin et al. 2003). Based on the cosmic $N(S)$ relation previously discussed, the majority of these are unlikely to be unrelated background sources. Presuming that the off-nuclear X-ray sources have distances as for the corresponding galaxy, we do not find any ULXs with

\footnotetext{
12 We have been awarded Cycle $19 H S T /$ ACS observations to verify that the incidence of nuclear star clusters within field early types is similar to that found for the AMUSE-Virgo survey.
}

$L_{\mathrm{X}} \gtrsim 10^{40} \mathrm{erg} \mathrm{s}^{-1}$. A complete catalog of ULXs for the Field snapshot galaxies will be presented in a later work, in which their properties will be placed in context with our upcoming HST observations and will be compared to ULXs found within the AMUSE-Virgo snapshot sample.

\subsubsection{X-Ray Emission from Stellar Tidal Disruption}

One interesting possibility for fueling SMBHs is through tidal disruption of stars (e.g., Rees 1988). Simulations indicate that such events could result in temporary super-Eddington infall, followed by thin-disk accretion, likely transitioning to a radiatively inefficient flow (Strubbe \& Quataert 2009). The predicted spectrum includes soft X-ray blackbody emission, plausibly with an additional power-law tail (Strubbe \& Quataert 2011). A handful of individual cases of potential tidal disruption events have been identified (e.g., Lin et al. 2011, and references therein), including most recently the spectacular Swift J164449.3+573451 (Burrows et al. 2011; Levan et al. 2011); in this rare case $L_{\mathrm{X}}$ significantly exceeds $L_{\mathrm{Edd}}$ for the inferred modest black hole mass $\left(\log M_{\mathrm{BH}} / M_{\odot}=5.5 \pm 1.1\right.$; Miller \& Gültekin 2011), suggesting that the observed emission comes predominantly from a beamed relativistic jet (Bloom et al. 2011; Burrows et al. 2011; Cannizzo et al. 2011). Deep surveys generally provide only loose upper limits on the typical rate (e.g., $<10^{-4} \mathrm{yr}^{-1}$ per galaxy; Luo et al. 2008), but numerical simulations carried out by Brockamp et al. (2011) suggest that approximately $3.0 \pm 1.4$ events per $10^{5} \mathrm{yr}$ are typical for an SMBH of $10^{6} M_{\odot}$. We use this rate to estimate the fraction of time for which a $10^{6} M_{\odot}$ black hole would display $L_{\mathrm{X} \text {,tidal }}>2.5 \times 10^{38} \mathrm{erg} \mathrm{s}^{-1}$ (the AMUSE-Field detection limit).

The timescale until the accretion rate drops below Eddington, following the canonical $t^{-5 / 3}$ scaling (Rees 1988), is $\lesssim 0.3-1 \mathrm{yr}$ (Strubbe \& Quataert 2009). Subsequent evolution of the diskdominated emission as $t^{-1.2}$ (Cannizzo et al. 1990) is likely curtailed after $\dot{m} \lesssim 10^{-2} m_{\text {Edd }}$ (Strubbe \& Quataert 2009), around $\sim 100 \mathrm{yr}$, after which material likely accretes through a thick disk. Presuming isotropic emission for simplicity, and taking $L_{\mathrm{X} \text {,tidal }} \simeq 10^{42} \mathrm{erg} \mathrm{s}^{-1}$ during the initial flare (motivated by Strubbe $\&$ Quataert 2011), $L_{\mathrm{X} \text {,tidal }} \simeq 4 \times 10^{39} \mathrm{erg} \mathrm{s}^{-1}$ after $\sim 100 \mathrm{yr}$, dropping below our detectability threshold by $\lesssim 1000$ yr (or sooner for an abrupt transition to radiatively inefficient accretion). The fraction of time for which a tidal disruption event could dominate the X-ray emission observed in a similar AMUSE-Field galaxy is then $\lesssim 3 \%$. (These estimates are for a solar-mass main-sequence star; see, e.g., Li et al. 2002 and Lodato et al. 2009 for alternatives). For reference, the AMUSE-Field sample contains 31 galaxies with $M_{\mathrm{BH}}<$ $10^{6} M_{\odot}$, so we might anticipate detecting X-ray emission associated with stellar disruption in about one object.

For SMBHs with $M_{\mathrm{BH}}>10^{7} M_{\odot}$, tidal disruption is expected to be less significant, as the distance at which a stellar trajectory can dynamically enter the loss cone exceeds the radius of influence (Brockamp et al. 2011), and in addition the refill timescale is quite long in large ellipticals, particularly core galaxies (Merritt \& Wang 2005). Most (42/52) of the AMUSEField galaxies with X-ray detections have $M_{\mathrm{BH}}>10^{7} M_{\odot}$. In view of the above, we conclude that the nuclear X-ray emission in the AMUSE-Field galaxies is not related to tidal disruption events, although without multi-epoch observations we cannot rule it out for any specific object. Instead, the SMBHs in these galaxies are more likely currently fed through quasicontinuous but highly sub-Eddington accretion, as arising from, 
Table 3

Sample Properties

\begin{tabular}{|c|c|c|c|c|c|c|c|c|c|c|c|}
\hline Sample & $n$ & Mean & 25 th & 50 th & 75 th & Sample & $n$ & Mean & 25 th & 50 th & 75 th \\
\hline \multicolumn{6}{|c|}{$\log \left(M_{\text {star }} / M_{\odot}\right)$} & \multicolumn{6}{|c|}{$M_{\mathrm{B}}(\mathrm{mag})$} \\
\hline Field & 103 & $9.66 \pm 0.12$ & 8.56 & 9.75 & 10.84 & Field & 103 & $-17.55 \pm 0.25$ & -19.82 & -17.64 & -15.39 \\
\hline Snapshot & 61 & $8.98 \pm 0.13$ & 8.02 & 8.71 & 9.96 & Snapshot & 61 & $-16.18 \pm 0.27$ & -17.84 & -15.59 & -14.21 \\
\hline Elliptical & 93 & $9.62 \pm 0.13$ & 8.44 & 9.71 & 10.74 & Elliptical & 93 & $-17.48 \pm 0.26$ & -19.73 & -17.41 & -15.22 \\
\hline In group & 74 & $9.86 \pm 0.15$ & 8.71 & 10.07 & 11.05 & In group & 74 & $-17.94 \pm 0.30$ & -20.15 & -18.21 & -15.36 \\
\hline \multicolumn{6}{|c|}{$\log \left(M_{\mathrm{BH}} / M_{\odot}\right)$} & \multicolumn{6}{|c|}{$\log L_{\mathrm{X}}$ (detections) } \\
\hline Field & 103 & $6.90 \pm 0.12$ & 5.75 & 6.79 & 7.96 & Field & 50 & $39.18 \pm 0.09$ & 38.65 & 39.00 & 39.40 \\
\hline Snapshot & 61 & $6.30 \pm 0.12$ & 5.50 & 6.08 & 7.08 & Snapshot & 19 & $38.87 \pm 0.09$ & 38.58 & 38.69 & 39.03 \\
\hline Elliptical & 93 & $6.86 \pm 0.12$ & 5.70 & 6.76 & 7.95 & Elliptical & 44 & $39.12 \pm 0.09$ & 38.64 & 38.96 & 39.35 \\
\hline In group & 74 & $7.08 \pm 0.15$ & 5.82 & 7.27 & 8.28 & In group & 38 & $39.22 \pm 0.10$ & 38.72 & 39.04 & 39.46 \\
\hline \multicolumn{6}{|c|}{$\log \left(M_{\mathrm{BH}} / M_{\mathrm{star}}\right)$} & \multicolumn{6}{|c|}{$\log \left(L_{X} / 10^{37}\right)(\text { all })^{\mathrm{a}}$} \\
\hline Field & 103 & $-2.76 \pm 0.04$ & -2.90 & -2.75 & -2.53 & Field & 97 & $1.71 \pm 0.07$ & 0.63 & 1.37 & 2.02 \\
\hline Snapshot & 61 & $-2.68 \pm 0.05$ & -2.82 & -2.68 & -2.48 & Snapshot & 61 & $1.42 \pm 0.05$ & 0.44 & 0.88 & 1.55 \\
\hline Elliptical & 93 & $-2.76 \pm 0.04$ & -2.89 & -2.75 & -2.55 & Elliptical & 87 & $1.67 \pm 0.07$ & 0.62 & 1.32 & 1.97 \\
\hline In group & 74 & $-2.77 \pm 0.05$ & -2.89 & -2.74 & -2.47 & In group & 68 & $1.78 \pm 0.08$ & 0.70 & 1.62 & 2.11 \\
\hline
\end{tabular}

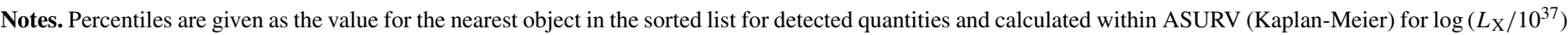

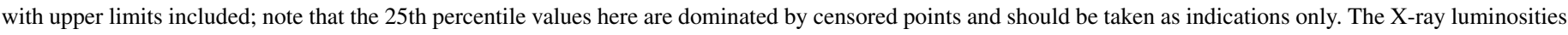
are restricted to $\log L_{\mathrm{X}}>38.2$ to avoid any potential bias from archival observations.

a Values are biased because the ASURV changed the first upper limit to a detection for the Kaplan-Meier computation.

for example, mass loss from evolved stars (Soria et al. 2006b; Volonteri et al. 2011).

\subsection{Stacking Non-detection}

The 42 snapshot observations lacking a nuclear X-ray detection were stacked to check for a joint detection and assess their average X-ray luminosity. After excising all off-nuclear X-ray point sources (occupying areas here defined by their wavdetect ellipses), the $0.3-7 \mathrm{keV}$ images were stacked at the optical position of each target. The combined image (Figure 1, lower right) has an effective exposure of $307 \mathrm{ks}$. There is a significant excess of counts near the center, with 42.2 net counts (87.1 total, 44.9 background) present within a 10 pixel (4".9) extraction radius. (Stacking random positions does not produce a significant detection.) For an average $N_{\mathrm{H}}$ of $2 \times 10^{20} \mathrm{~cm}^{-2}$ and taking $\Gamma=2$, the rate of $(1.4 \pm 0.4) \times 10^{-4}$ counts s ${ }^{-1}$ corresponds to an average (unabsorbed) $0.3-10 \mathrm{keV}$ flux of $1.1 \times 10^{-15} \mathrm{erg}$ $\mathrm{s} \mathrm{cm}^{-2}$. The mean distance modulus for these 42 objects is $31.5(20.0 \mathrm{Mpc})$, which gives an average X-ray luminosity of $\log L_{\mathrm{X}}=37.7 \mathrm{erg} \mathrm{s}^{-1}$. Given the total enclosed stellar mass, this is broadly consistent with arising from a population of individually undetectable LMXBs.

The excess X-ray emission within the stacked image is centrally concentrated but not pointlike and extends to $\gtrsim 20^{\prime \prime}$. The net rates within apertures of 4, 6, 10, 20, 40, and 60 pixels

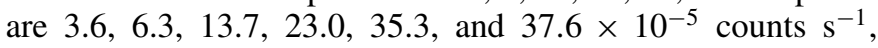
respectively. This indicates that the excess $\mathrm{X}$-ray emission is not exclusively nuclear and likely includes contributions from off-nuclear LMXBs as well as hot gas.

These results further confirm that the nuclear X-ray luminosities calculated for snapshot targets with X-ray detections do not suffer significantly from LMXB or hot gas contamination. With the caveat that the presence or absence of a nuclear star cluster has not been established for specific sources, the average overall contribution to $L_{\mathrm{X}}$ from an underlying ensemble of LMXBs within a $2^{\prime \prime}-3^{\prime \prime}$ aperture based on the above measurement is $<5 \%-9 \%$ of the minimum snapshot detected nuclear $\mathrm{X}$-ray emission $\left(\log L_{\mathrm{X}}=38.4 \mathrm{erg} \mathrm{s}^{-1}\right)$, and the contribution from hot gas is negligible.

\section{GLOBAL PROPERTIES OF THE AMUSE-FIELD SAMPLE}

Here, we determine and discuss the global properties of the AMUSE-Field sample, including stellar and black hole masses as well as Eddington-scaled X-ray luminosities. We then proceed to investigate the scaling between the measured nuclear X-ray luminosity and the host galaxy stellar mass, and we calculate the residual X-ray luminosity after accounting for this dependence. Finally, we test against the full Field sample the properties of the snapshot targets (which constitute a uniform-sensitivity subset) and of elliptical galaxies (to check for possible systematic morphological effects).

Table 3 contains the derived properties of the full AMUSEField sample as well as three subsets thereof, composed of (1) snapshot targets, (2) elliptical galaxies, and (3) group members. (The influence of group richness is discussed in more detail in Section 5.) For each of the above subsets, along with the full sample, the mean and 25th, 50th, and 75th percentiles are calculated for the following quantities: $\log \left(M_{\text {star }} / M_{\odot}\right)$, $\log \left(M_{\mathrm{BH}} / M_{\odot}\right), \log \left(M_{\mathrm{BH}} / M_{\mathrm{star}}\right), M_{\mathrm{B}}$, and $\log L_{\mathrm{X}}$ for both detections and for all objects. The Kaplan-Meier distribution, which handles censored data (Kaplan \& Meier 1968), was determined for X-ray luminosities including upper limits using the survival analysis package ASURV ${ }^{13}$ (e.g., Lavalley et al. 1992). ${ }^{14}$ To reduce any potential bias from archival observations, we conservatively impose a luminosity limit of $\log L_{\mathrm{X}}>38.2$ when examining the distribution of $\log L_{\mathrm{X}}$ (or quantities derived therefrom) with limits included and when carrying out fitting of $L_{\mathrm{X}}\left(M_{\text {star }}\right)$; six objects (two detected) from the full Field sample do not meet this screening criterion. The adopted methodology and calculation of these quantities are described in detail in the following subsections.

\footnotetext{
13 http://astrostatistics.psu.edu/statcodes/asurv

14 Note, however, that at least the 25 th percentile values are dominated by upper limits, and the mean values are biased owing to the conversion of a limit to a detection for calculation purposes, so these values should be taken as roughly indicative only.
} 


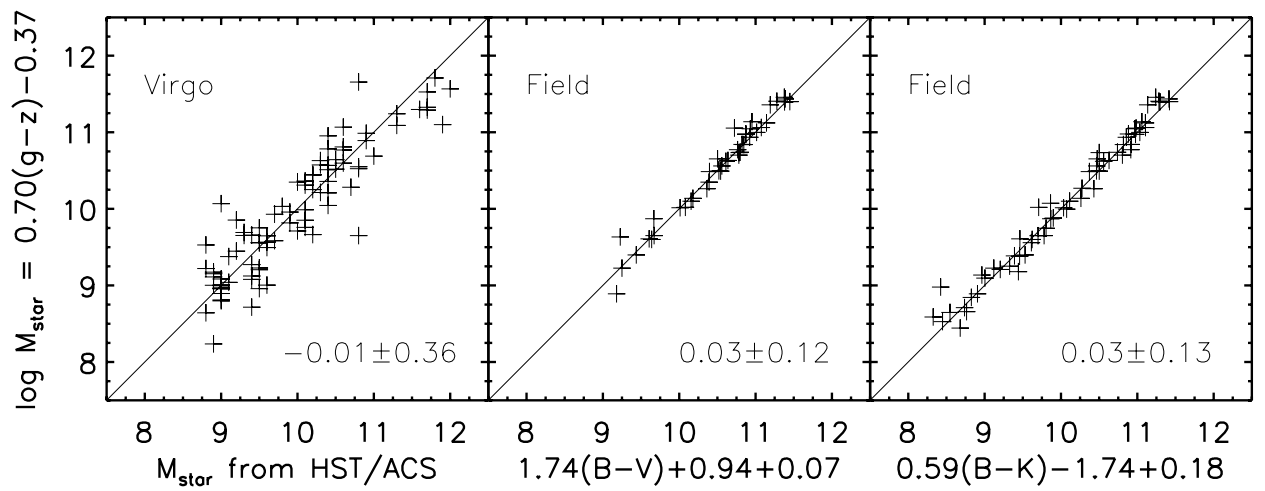

Figure 3. Illustration of methodology used to calculate host galaxy stellar mass, $M_{\text {star }}$. For the Field sample, $M_{\text {star }}$ is calculated from absolute $B$ magnitude and either $g-z, B-V$, or $B-K$ color, in that order of precedence; see Section 4.1 for details. After adjustment to the median offset, the different colors give consistent $M_{\text {star }}$.

\subsection{Stellar and Black Hole Masses}

The host galaxy stellar mass is calculated from the absolute $B$ magnitude and from optical/IR color. Absolute $B$ magnitudes for the total galaxy (for these sources, essentially equivalent to the spheroidal luminosities) are taken from the HyperLeda catalog. These are based on total $B$ magnitudes reduced to the RC3 system and corrected for Galactic absorption, and calculated for distances identical to those used to determine $L_{X}$. We calibrate our procedure against the stellar masses established for the galaxies in the AMUSE-Virgo survey, which were determined based on F457W and F850LP HST/ACS imaging (G08, Section 3.3); these filters roughly correspond to SDSS $g$ and $z$ bands (Fukugita et al. 1996) and provide nearly equivalent colors. The primary relation used to calculate stellar mass is $M_{\text {star }}=$ $0.70 \times(g-z)-0.37+0.4 \times\left(5.45-M_{B}\right)$ (based on Table 7 of Bell et al. 2003); Figure 3 illustrates that using SDSS $g-z$ model-magnitude colors (corrected for Galactic extinction) gives results consistent with those obtained from the higher-resolution $H S T /$ ACS data, with a mean difference of $-0.01 \pm 0.36$. Where SDSS coverage is not available, we use instead corrected $B-V$ colors (taken from HyperLeda) as $M_{\text {star }}=1.74 \times(B-V)+0.94+0.4 \times\left(5.45-M_{B}\right)$ (based on Table 7 of Bell et al. 2003), but here we also add 0.07, which is the empirical median offset between $M_{\text {star }}$ derived from $g-z$ versus $B-V$ colors. No physical meaning is ascribed to this adjustment, which may merely arise from inconsistent effective apertures; similar median offsets are found for AMUSEVirgo galaxies. The agreement between these methods is good (Figure 3; mean difference $0.03 \pm 0.12$ ). Where neither SDSS coverage nor HyperLeda $B-V$ magnitudes are available, we use $B-K$ colors (with total $K$ magnitudes taken from the Two Micron All Sky Survey (2MASS) catalog of extended sources) as $M_{\text {star }}=0.59 \times(B-K)-1.74+0.4 \times\left(5.45-M_{B}\right)($ based on Table 1 of Bell \& de Jong 2001), but here we also add 0.18, which is the empirical median offset between $M_{\text {star }}$ derived from $g-z$ versus $B-K$ colors. The agreement between the $g-z$ and $B-K$ methods is also good (Figure 3 ; mean difference $0.03 \pm 0.13$ ). Finally, in the four cases (PGC 132768, PGC 064718, PGC 740586, and 6dF J2049400-324154) where neither SDSS coverage, nor HyperLeda $B-V$ colors, nor 2MASS extended-source $K$ magnitudes were available, the median $g-z=1.16$ is used to estimate $M_{\text {star }}$. The X-ray detection fraction for the Field sample is illustrated versus $M_{\text {star }}$ in Figure 4.

Black hole masses $\left(M_{\mathrm{BH}}\right)$ are estimated from the mass-stellarvelocity dispersion $\left(M_{\mathrm{BH}}-\sigma\right)$ or else from the mass-bulgeluminosity $\left(M_{\mathrm{BH}}-L_{B}\right)$ correlations (e.g., see overview by

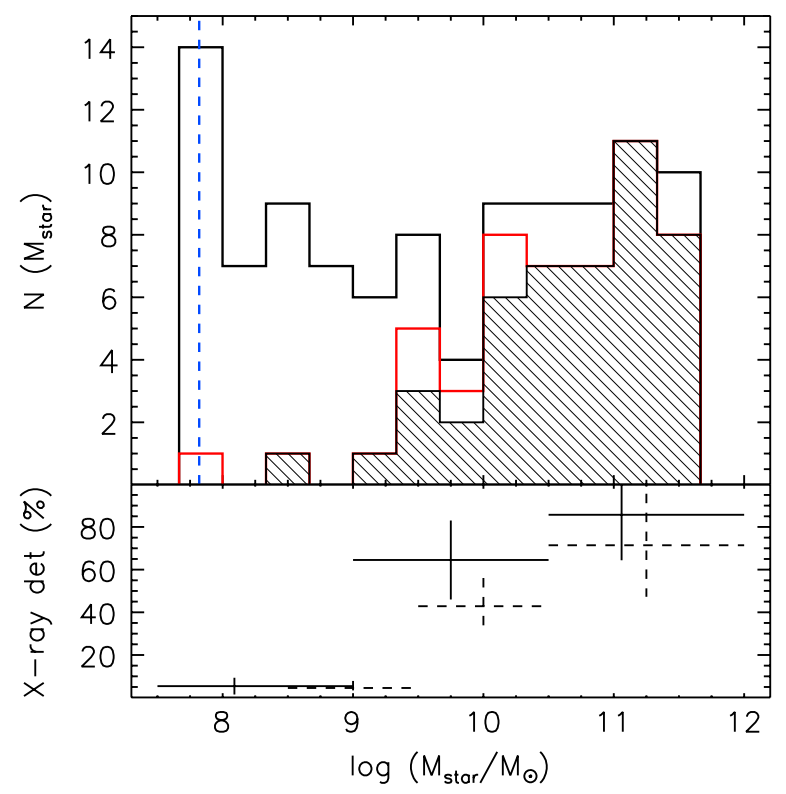

Figure 4. Top panel shows the distribution of stellar mass within the Field sample. The red histogram shows nuclear X-ray detections, while the shaded histogram incorporates a statistical correction for potential LMXB contamination from nuclear star clusters. The bottom panel shows the X-ray detection fraction (solid crosses) within bins of low, moderate, and high stellar mass. The increased detection fraction with increasing stellar mass (e.g., Ho et al. 1997) results here primarily from an ability to detect X-ray emission to a lower Eddington fraction in higher-mass objects. A qualitatively similar effect was found for the Virgo sample (dashed crosses in bottom panel).

(A color version of this figure is available in the online journal.)

Ferrarese \& Ford 2005). These have been calibrated with independent measures of $M_{\mathrm{BH}}$, such as yielded by reverberation mapping (Woo et al. 2010), enabling straightforward estimation of $M_{\mathrm{BH}}$. We use the updated relations presented in Gültekin et al. (2009), specifically those derived for early-type galaxies. As they find the scatter in the $M_{\mathrm{BH}^{-}}-\sigma$ relation to be slightly lower than in the $M_{\mathrm{BH}}-L$ relation for early-type galaxies, we use the former where high-quality measurements of $\sigma$ are available in the literature. We note that caution is warranted when applying this correlation to a heterogeneous sample, as it is not clear that the $M_{\mathrm{BH}}-\sigma$ relation holds in identical form to low masses (Greene et al. 2010) or high luminosities (Lauer et al. 2007) or for barred galaxies (Graham 2008) or to higher redshifts (Treu et al. 2007; Woo et al. 2008). Only the first of these points is potentially relevant for this sample, and in practice there is only one Field galaxy with $\sigma<25 \mathrm{~km} \mathrm{~s}^{-1}$, a measurement we discard owing to concerns as to systematic errors. 

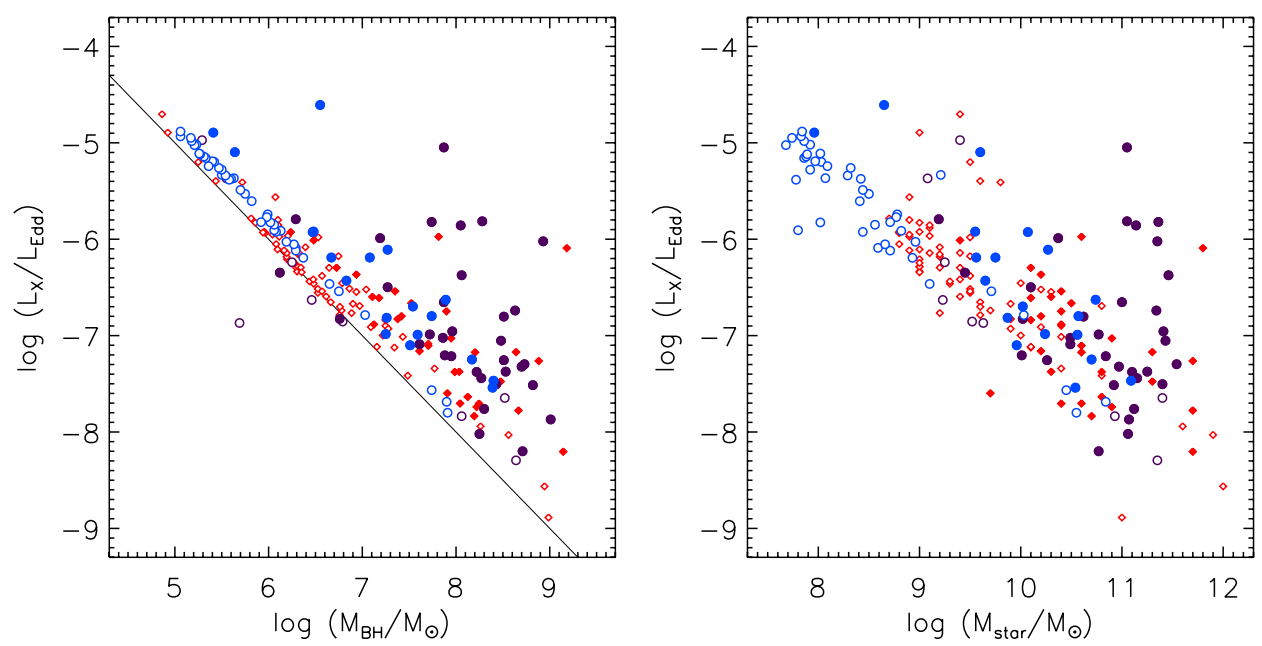

Figure 5. Ratio of X-ray to Eddington luminosity as a function of black hole mass (left panel) and stellar mass (right panel). The Field sample is plotted with circles (snapshot/archival sources are blue/purple) and the Virgo sample is also plotted, with red diamonds, for comparison. X-ray detections/limits are shown as filled/open symbols. The solid black line in the left panel indicates the sensitivity limit of the Virgo survey, corresponding to the Eddington limit for a one solar mass black hole. While the distributions of black hole and stellar mass are formally inconsistent between the field and Virgo samples, qualitatively similar trends with Eddington-scaled $\mathrm{X}$-ray luminosity are observed.

(A color version of this figure is available in the online journal.)

Eddington luminosities are calculated from the abovedetermined black hole mass as $L_{\text {Edd }}=1.3 \times$ $10^{38}\left(M_{\mathrm{BH}} / M_{\odot}\right) \mathrm{erg} \mathrm{s}^{-1}$, and Eddington-scaled X-ray luminosities, $\log \left(L_{\mathrm{X}} / L_{\mathrm{Edd}}\right)$, for the AMUSE-Field sample are plotted in Figure 5 versus both $M_{\mathrm{BH}}$ and $M_{\mathrm{star}}$. Values from the AMUSEVirgo sample are also shown for comparison. The distributions of $M_{\mathrm{BH}}$ and $M_{\text {star }}$ are formally inconsistent (K-S test $p=0.01$ and $p<0.001$, respectively) between the Field and Virgo samples, but qualitatively similar general trends with Eddingtonscaled X-ray luminosities are observed.

It appears that a fixed $L_{\mathrm{X}} / L_{\mathrm{Edd}}$ value (or range) across $5<\log \left(M_{\mathrm{BH}} / M_{\odot}\right)<9$ does not provide an adequate match to the observed data, which show a scarcity of large $L_{\mathrm{X}} / L_{\mathrm{Edd}}$ values at large $M_{\mathrm{BH}}$. All objects are highly sub-Eddington, with Eddington-scaled nuclear X-ray luminosities ranging from $<10^{-8}$ to $10^{-4}$. Clearly, the limiting sensitivity of the survey $\left(\sim 2.5 \times 10^{38} \mathrm{erg} \mathrm{s}^{-1}\right.$ for snapshot targets) acts to restrict the observable Eddington ratio for detected sources: because of the correlation between black hole mass and host stellar mass (linked to the underlying dependence of stellar mass on bulge optical luminosity), nuclear X-ray sources may be detected down to lower Eddington-scaled luminosities within higher $M_{\text {star }}$ galaxies, and this Eddington-incompleteness effect can explain the increase in the X-ray detection fraction with increasing stellar mass seen in Figure 4. This effect was noted by G10 for the Virgo sample. Despite these observational complications, the relative weakness of SMBH activity associated with the highest black hole masses in the Field sample is a qualitatively similar trend to the "downsizing" tendency noted by G10.

\subsection{Nuclear X-Ray Luminosity as a Function of Host Stellar Mass}

In the following we assess the relationship between nuclear $\mathrm{X}$-ray luminosity and stellar mass within the AMUSE-Field sample to investigate how activity in the nucleus is linked to the large-scale properties of the host galaxy. As $M_{\text {star }}$ is calculated from $M_{\mathrm{B}}$ as well as color, this analysis is similar to examining a luminosity-luminosity correlation and is grounded in observed quantities. It is reasonable to expect that some factor of the observed correlation between nuclear X-ray luminosity and stellar mass is merely reflective of a correlation between nuclear X-ray luminosity and black hole mass; because $M_{\text {star }}$ and $M_{\mathrm{BH}}$ are correlated and, for many of these objects, both based on $M_{\mathrm{B}}$, it is difficult to disentangle their relative influence on nuclear $\mathrm{X}$-ray luminosities. Here we restrict out analysis to nuclear $\mathrm{X}$-ray luminosity considered as a function of host stellar mass; we defer study of the $L_{\mathrm{X}}\left(M_{\mathrm{BH}}\right)$ relation to a later work (Miller et al. 2012), in which we compare the functional form of this relation within the AMUSE-Field sample with that found for the AMUSE-Virgo cluster early types (G10), while fully accounting for the multiple methods of determining $M_{\mathrm{BH}}$ and for the inconsistency in $M_{\text {star }}$ distributions between the Field and Virgo samples.

Fitting is carried out using the Bayesian IDL code of Kelly (2007), which incorporates both uncertainties and censoring, to determine the best-fit parameters. The functional form investigated is $\left(\log L_{\mathrm{X}}-38.4\right)=A+B \times\left(\log M_{\text {star }}-9.8\right)$, where the variables are centered on approximately the median values. Points with $\log L_{\mathrm{X}}<38.2$ are excluded to avoid any potential bias from archival observations, but this has only a minimal effect on the result (e.g., the slope is insignificantly flattened by $0.07 \pm 0.14$ ). Errors are taken to be 0.1 on $\log L_{\mathrm{X}}$ and 0.1 on $\log \left(M_{\text {star }} / M_{\odot}\right)$, in both cases associated with the uncertainty in the distance rather than measurement error of the X-ray counts or the bulge luminosity. Several alternative forms of errors were investigated, including errors increasing with decreasing luminosities; in general, larger errors produce a slightly steeper slope but do not appreciably affect any of the below conclusions. Three Gaussians are used in the independent variable mixture modeling, and a minimum of 5000 iterations are performed with Gibbs sampling. The most likely parameter values are estimated as the median of 10,000 draws from the posterior distribution, with credible intervals corresponding to $1 \sigma$ errors calculated as the 16 th and 84th percentiles. For completeness, we verified that using instead ASURV with either the expectation maximization or Buckley-James method (Buckley \& James 1979) identifies similar trends, with slightly flatter slopes.

Results are illustrated in Figure 6, with best-fit coefficients listed in Table 4. For the full AMUSE-Field sample, with all objects included, the best-fit relation is $\left(\log L_{X}-38.4\right)=$ 
Table 4

Correlation between Nuclear X-Ray Luminosity and Stellar Mass

\begin{tabular}{|c|c|c|c|c|c|c|c|c|}
\hline \multirow[t]{2}{*}{ Sample } & \multicolumn{4}{|c|}{ All Data } & \multicolumn{4}{|c|}{ Detections } \\
\hline & $n$ & $A$ & $B$ & $\sigma$ & $n$ & $A$ & $B$ & $\sigma$ \\
\hline Field & 97 & $-0.04_{-0.12}^{+0.11}$ & $0.71_{-0.09}^{+0.10}$ & $0.73_{-0.08}^{+0.10}$ & 50 & $0.52_{-0.12}^{+0.13}$ & $0.35_{-0.12}^{+0.11}$ & $0.59_{-0.06}^{+0.07}$ \\
\hline Snapshot & 61 & $-0.13_{-0.18}^{+0.14}$ & $0.66_{-0.15}^{+0.19}$ & $0.74_{-0.13}^{+0.17}$ & 19 & $0.47_{-0.10}^{+0.11}$ & $0.00_{-0.14}^{+0.15}$ & $0.43_{-0.08}^{+0.10}$ \\
\hline Elliptical & 87 & $-0.03_{-0.11}^{+0.10}$ & $0.65_{-0.08}^{+0.09}$ & $0.71_{-0.08}^{+0.09}$ & 44 & $0.51_{-0.11}^{+0.11}$ & $0.30_{-0.11}^{+0.11}$ & $0.54_{-0.06}^{+0.07}$ \\
\hline In group & 68 & $-0.07_{-0.13}^{+0.13}$ & $0.72_{-0.11}^{+0.11}$ & $0.67_{-0.08}^{+0.10}$ & 38 & $0.43_{-0.15}^{+0.15}$ & $0.43_{-0.13}^{+0.13}$ & $0.54_{-0.06}^{+0.08}$ \\
\hline
\end{tabular}

Notes. Fits are to the function $\left(\log L_{\mathrm{X}}-38.4\right)=A+B \times\left(\log M_{\mathrm{star}}-9.8\right)$, where $\sigma$ is the intrinsic scatter. Fitting is carried out using the IDL code of Kelly (2007); see Section 4.2 for details.

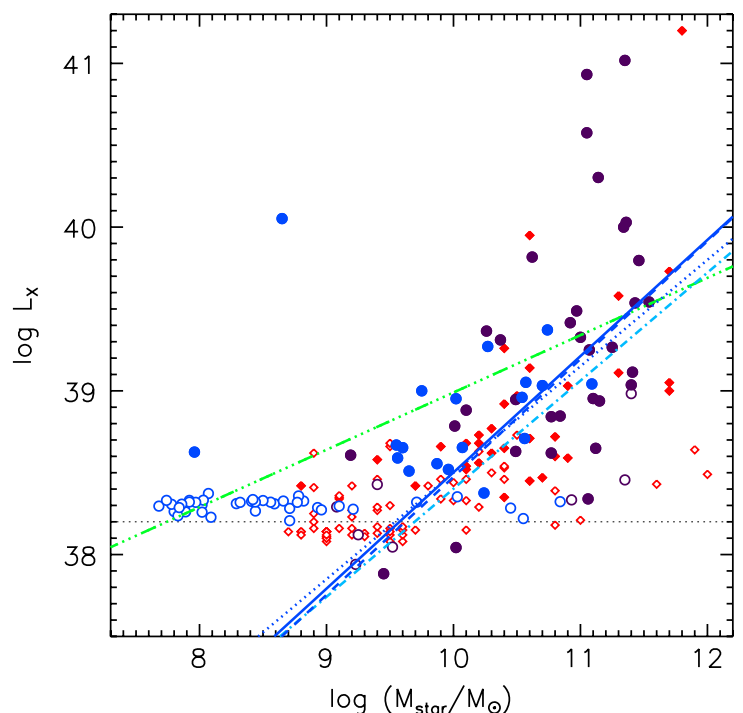

Figure 6. X-ray luminosity as a function of host galaxy stellar mass. Symbols are as in Figure 5. The solid line is the best-fit linear relation (to logarithmic quantities, for objects with $\log L_{X}>38.2$ ) for the full Field sample, while the dot-dashed, dotted, and dashed lines are for the snapshot, elliptical, and group member subsets; see Section 4.2 for details. For reference, the triple-dot-dashed line is the fit to X-ray detections only. All fit coefficients are provided in Table 4. (A color version of this figure is available in the online journal.)

$(-0.04 \pm 0.12)+(0.71 \pm 0.10) \times\left(\log M_{\text {star }}-9.8\right)$, with a rather large intrinsic scatter of $\sigma=0.73 \pm 0.09$. Consistent results are obtained for the subsets of snapshot targets, ellipticals, and group members. If only X-ray-detected points are considered, the slope is considerably flatter, $B=0.35 \pm 0.12$ for the full sample. Residual X-ray luminosities may now be calculated as $\Delta L_{\mathrm{X}, \mathrm{M} *}=\log L_{\mathrm{X}}-\log L_{\mathrm{X}}\left(\log M_{\text {star }}\right)$, where $\log L_{\mathrm{X}}\left(\log M_{\text {star }}\right)$ is the best-fit linear relation for the full Field sample as defined above. $\Delta L_{\mathrm{X}, \mathrm{M} *}$ thus provides a measure of relative $\mathrm{X}$-ray brightness after accounting for the typical influence of stellar mass.

As $M_{\text {star }}$ is derived from absolute $B$ luminosity, the correlation we find for $L_{\mathrm{X}}$ as a function of $M_{\text {star }}$ is qualitatively similar to the tendency noted by Pellegrini (2010) for $L_{\mathrm{X}}$ to increase with $L_{\mathrm{B}}$. The slope for the full AMUSE-Field sample of $B=0.71 \pm 0.10$ is incompatible with zero dependence but also appears to be less than unity (at the $2.9 \sigma$ level), indicating that nuclear X-ray luminosity may increase less rapidly than $M_{\text {star }}$ : this would imply that lower-mass galaxies are more X-ray luminous per unit stellar mass. While the general trend in Figure 6 is clear, the observed dispersion in both $L_{\mathrm{X}}$ and $\Delta L_{\mathrm{X}, \mathrm{M} *}$ is larger for $\log M_{\text {star }}>10.5 M_{\odot}$, and so it is possible that a more complex model could provide an improved representation of $L_{X}$ across the full range of stellar masses.

\subsection{Uniform Sensitivity and Morphologically Distinct Sub-samples}

While the AMUSE-Field sample is by design fairly homogeneous, it does include both snapshot and archival objects and both type E and type E-S0 galaxies. The snapshot sample possesses an approximately constant X-ray luminosity sensitivity threshold due to the deliberate choice of exposure times, which is not the case for the archival sample. Elliptical galaxies are completely spheroidal, whereas transition objects may include small disk components. It is thus useful to check the characteristics of these subsets.

Snapshot targets. The archival Chandra coverage available prior to selection of the snapshot sample understandably favored brighter galaxies expected to provide a high X-ray count rate. Within our volume-limited sample, the snapshot targets therefore tend to be galaxies of lower optical luminosity and lower stellar mass. The ratios of black hole to stellar mass [i.e., $\left.\log \left(M_{\mathrm{BH}} / M_{\text {star }}\right)\right]$ are consistent with each other $(\mathrm{K}-\mathrm{S} p=0.29)$ between the snapshot targets and the full Field sample, as $\log M_{\mathrm{BH}}$ is also lower for the snapshot targets. (Note that for a majority of the snapshot targets the $M_{\mathrm{BH}}-L$ relation is used to calculate $M_{\mathrm{BH}}$ and so black hole mass is directly dependent on optical luminosity. The $M_{\mathrm{BH}}-L$ relation predicts slightly higher $M_{\mathrm{BH}}$ values at low luminosities; see, e.g., discussion in G08.) The snapshot targets tend toward modestly lower Xray luminosities where detected, and the difference is enhanced when upper limits are included (owing to the lower detection fraction for the snapshot targets, Section 3.1); the snapshot targets have lower mean and Kaplan-Meier quartile $\log L_{\mathrm{X}}$ values, and two-sample tests conducted within ASURV indicate that the snapshot distribution of $\log L_{X}$ is inconsistent with that of the full Field sample (logrank $p=0.006$, Peto \& Prentice $p=0.003$; Latta 1981). However, the best-fit $\log L_{\mathrm{X}}\left(\log M_{\text {star }}\right)$ relation for snapshot targets has an intercept and slope consistent with that for the full Field sample. Further, the distribution of residual X-ray luminosities for the snapshot targets matches that of the full Field sample; the $\Delta L_{\mathrm{X}, \mathrm{M} *}$ Kaplan-Meier quartiles are $-0.29,0.07,0.37$ for the snapshot targets and $-0.36,0.05$, 0.38 for the Field sample, and two-sample tests also support consistency (logrank and Peto \& Prentice $p>0.5$ ). This confirms that after accounting for the dependence of $L_{\mathrm{X}}$ on $M_{\text {star }}$, the snapshot targets have X-ray properties in line with those of the full Field sample. As the snapshot observations are sensitive to a uniform limiting luminosity (Section 2), we further conclude that the full Field sample is not biased by the wide variety in exposure times of the archival objects.

Elliptical galaxies. Most $(93 / 103)$ of the galaxies in the Field sample are ellipticals, and so it is not surprising that the properties of these ellipticals are virtually identical to those 
of the full Field sample. The detection fraction for ellipticals is $46 / 93$ or $49 \%$, whereas for non-ellipticals (including three somewhat irregular spheroids unclassified in HyperLeda) it is $6 / 10$ or $60 \%$ (for E-S0 galaxies in particular it is 6/7). While the mean or median $L_{\mathrm{X}}$ values for objects are $\sim 0.5$ dex greater for the 6 X-ray-detected type E-S0 galaxies than for the $46 \mathrm{X}$-raydetected type E galaxies, the same holds true for $M_{\text {star }}$ values, and so the mean and median $\Delta L_{\mathrm{X}, \mathrm{M} *}$ agree to within 0.1 . In any event the scatter is sufficiently large that both the $\log L_{\mathrm{X}}$ and $\Delta L_{\mathrm{X}, \mathrm{M} *}$ distributions for detected objects are consistent (K-S $p>0.5)$ between field type E and type E-S0 galaxies; more quantitative assessment of any minor differences in nuclear X-ray properties would require a much larger sample of type E-S0 galaxies. It would also be interesting to examine a companion sample of S0 galaxies, for which we might expect the active fraction and the typical nuclear X-ray luminosity to be somewhat higher than for ellipticals, based on the increasing AGN fraction toward later morphological types and bluer galaxies found by Choi et al. (2009) and attributed by them to a relatively greater gas supply.

\section{INFLUENCE OF GROUP RICHNESS ON NUCLEAR ACTIVITY}

Although by construction the galaxies in the AMUSE-Field sample do not reside in clusters (recall from Section 2 that the coordinate selection excluded objects in the direction of Fornax and Virgo), some of them are members of groups, inhabiting regions characterized by galaxy overdensities intermediate between isolated and cluster environments. Groups provide a suitable environment for strong interactions and mergers, since the number density is higher than for the field but the dispersion in radial velocities of galaxies in groups is smaller than that found in clusters (recall that the dynamical friction in a close encounter between two galaxies scales inversely with the square of their relative velocities). Tidal and ram-pressure stripping become increasingly important effects in richer groups (and clusters). As a consequence, membership in a group, and the richness of that group, could have an impact on the interaction history of a galaxy and on its gas content, both of which may be potentially relevant to the fueling of the SMBH. Here we investigate whether group membership or group properties influence nuclear X-ray activity.

We use the newly available catalog by Makarov \& Karachentsev (2011) to determine group membership, if any, for each target. There are eight AMUSE-Field objects not present in this catalog, and so the following analysis is limited to $95 \mathrm{ob}-$ jects. Following their approach, groups consist of four or more associated galaxies present within the zero-velocity surface; 74 $(78 \%)$ AMUSE-Field galaxies satisfy this criterion. The largest group included here is that centered on NGC 5846, which contains 74 members; the Field sample includes 18 of these, of which 3 have X-ray detections (the nucleus of NGC 5846 itself is not significantly detected above the diffuse emission; see the Appendix). Overall, the detection fraction for group members is $40 / 74$ or $54 \%$, while for objects with $n_{\text {group }}<4$ (we will refer to these as "non-group" members, but it is understood that they are also obviously not cluster members) it is $11 / 21$ or $52 \%$; these rates are consistent with being identical. There are 12 "isolated" galaxies (with $n_{\text {group }}=1$, although these may not be true void galaxies as in, e.g., Kreckel et al. 2011), of which 5 have X-ray detections or $42 \%$.

Restricting consideration to X-ray-detected objects, the mean or median $L_{\mathrm{X}}$ values for non-group members are consistent with those for group members (difference $<0.1 \mathrm{dex}$ ), but as the $M_{\text {star }}$ values of the non-group members tend to be lower by $\simeq 0.6 \mathrm{dex}$, their mean and median $\Delta L_{\mathrm{X}, \mathrm{M} *}$ values are higher (by $\simeq 0.4$ and $\simeq 0.3$, respectively). The scatter is large enough that the distributions of $\log L_{\mathrm{X}}$ and $\Delta L_{\mathrm{X}, \mathrm{M} *}$ are consistent (K-S $p>0.2)$ between group members or non-members. The five X-ray-detected-isolated ${ }^{15}$ galaxies have even lower $M_{\text {star }}$ values than other non-group members, and consequently higher $\Delta L_{\mathrm{X}, \mathrm{M} *}$ values (overcoming marginally lower $L_{\mathrm{X}}$ values); here again the distributions of $\log L_{\mathrm{X}}$ and $\Delta L_{\mathrm{X}, \mathrm{M} *}$ are not formally inconsistent at the $\alpha=0.05$ level (K-S $p=0.38$ and $p=0.06$, respectively; the latter case is perhaps borderline).

When upper limits are incorporated, similar conclusions hold. Comparing non-group to group members, the distributions in $\log L_{\mathrm{X}}$ and $\Delta L_{\mathrm{X}, \mathrm{M} *}$ are consistent (logrank and Peto \& Prentice $p>0.4)$, despite slightly lower $\log L_{X}$ and slightly higher $\Delta L_{\mathrm{X}, \mathrm{M} *}$ values (by $\sim 0.2 \mathrm{dex}$ ) in non-group members. The Kaplan-Meier quartiles are systematically somewhat lower in $\log L_{\mathrm{X}}$ for isolated galaxies compared with group members $(0.54,1.08,1.61$ versus $0.70,1.62,2.11)$, but somewhat greater in $\Delta L_{\mathrm{X}, \mathrm{M} *}(0.06,0.32,0.47$ versus $-0.40,0.03,0.30)$. The distributions are not formally inconsistent (logrank and Peto \& Prentice $p>0.1$ ). Eddington-scaled X-ray luminosities also display an apparent progression from lower to higher values moving from group to non-group to isolated galaxies, but again the significance is borderline. ${ }^{16}$

We conclude that there is suggestive but inconclusive evidence that non-group members, particularly isolated galaxies, tend toward slightly enhanced values of $\log L_{\mathrm{X}} / L_{\mathrm{Edd}}$ or $\Delta L_{\mathrm{X}, \mathrm{M} *}$ as compared with group members. If these results are confirmed, a possible interpretation is that interactions with the intragroup medium and other group members act to remove gas that could otherwise become available for SMBH accretion. This may be a secondary effect; for example, if star formation near the galaxy center were reduced by environmental interactions, less mass would be provided to the SMBH from stellar winds.

The nuclear X-ray luminosity, Eddington-scaled X-ray luminosity, and residual X-ray luminosity are plotted versus group size and group radial velocity dispersion in Figure 7. We also include the 100 cluster spheroids from the AMUSE-Virgo sample (G08, G10), with artificial random scatter added to their abscissa coordinates for improved visual clarity. The stellar mass of each galaxy is indicated by the size of the associated marker. While no strong trends are present and the scatter in X-ray properties is large, there appears to be a progression from generally higher to generally lower values of Eddington-scaled and residual X-ray luminosity moving from isolated to group to cluster environments. As groups are more hospitable to strong galaxy-galaxy interactions than are clusters (owing to lower galaxy velocity dispersions), a continuous progression implies that merger history may not be the dominant factor controlling current nuclear $\mathrm{X}$-ray activity. Instead, interactions with the intragroup or intracluster medium, which increase in importance as the galaxy velocity dispersions and the density of the medium increase, may influence low-level SMBH fueling. (A more quantitative

\footnotetext{
15 We note that ESO 540-014, isolated by these criteria, is an X-ray bright outlier.

16 Including upper limits, the mean $\log L_{\mathrm{X}} / L_{\text {Edd }}$ values for group members, non-group members, and isolated galaxies are $-7.06 \pm 0.11,-6.62 \pm 0.20$, and $-6.26 \pm 0.19$, with median values of $-7.22,-6.98$, and -6.63 ; removing ESO 540-014 from the isolated subset gives instead a mean of $-6.41 \pm 0.14$ and median of -6.69 . Two-sample tests for group members versus non-group members, isolated galaxies, or isolated galaxies excluding ESO 540-014 give logrank $p=0.10,0.01,0.05$ and Peto \& Prentice $p=0.10,0.03,0.12$.
} 


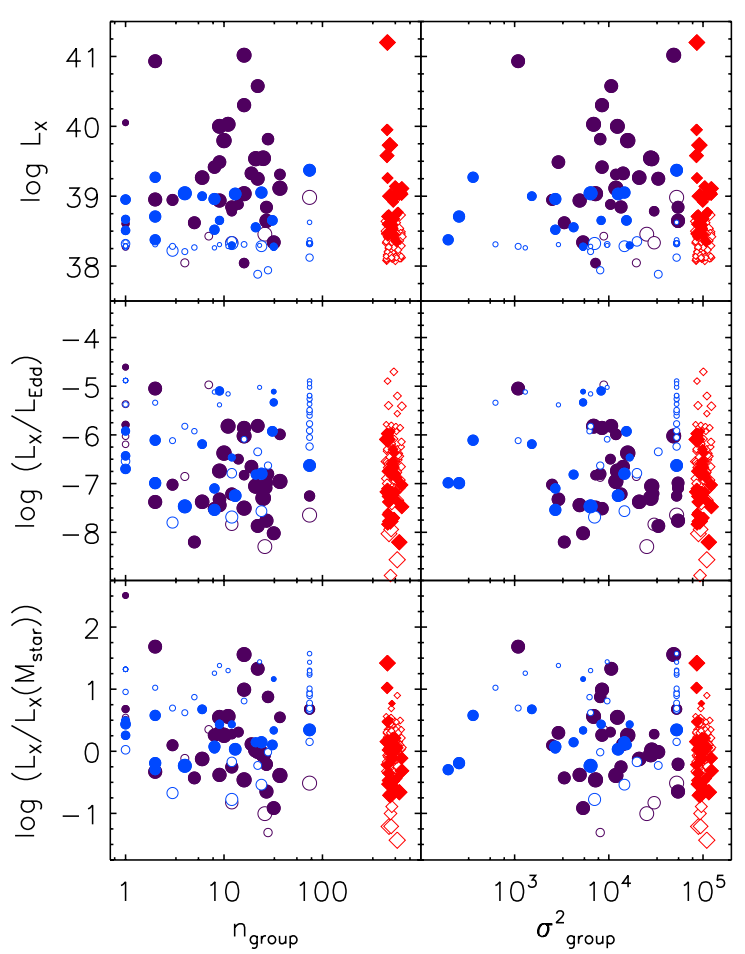

Figure 7. Nuclear X-ray luminosity (top), Eddington-scaled X-ray luminosity (middle), and residual X-ray luminosity (bottom) plotted as a function of group size (left) and group velocity dispersion (right; note plots truncate lower values of $\sigma_{\text {group }}^{2}$ ). Symbols are as in Figure 5 except that symbol size is here indicative of stellar mass. Artificial $x$-axis scatter has been added to the Virgo points to improve visual clarity.

(A color version of this figure is available in the online journal.)

and statistically robust comparison of X-ray luminosities in field versus cluster environments is provided in Miller et al. 2012).

Previous works examining the role of environment on X-ray emission in early-type galaxies found a trend opposite to that discussed above. A study using ROSAT data carried out by Brown \& Bregman (2000) concluded that early types in richer environments tended to be more $\mathrm{X}$-ray luminous, with a positive correlation between $L_{X} / L_{B}$ and galaxy density (but see also O'Sullivan et al. 2001). They speculated that this could reflect the influence of the hot intergalactic gas, either through acting to stifle galactic winds or more directly as an accretion source. We see no evidence for such a trend in the AMUSE-Field nuclear $\mathrm{X}$-ray luminosities. This discrepancy is almost certainly due to the much finer spatial resolution of Chandra, which enables us to disentangle nuclear, accretion-powered X-ray emission from soft diffuse X-ray emission from gas. In support of this conclusion, we note that the above ROSAT sample included several galaxies that are also members of our archival sample (NGC 0720, 1407, 4125, 4278, 4494, 4636, 4697, 5322, 5846) and that the X-ray luminosities measured by ROSAT are generally 1-2 orders of magnitude larger than those measured by Chandra.

\section{SUMMARY}

This work presents first results from the AMUSE-Field survey, a Large Chandra program designed to characterize low-level SMBH activity within nearby field spheroids. The Field sample contains 103 early-type non-cluster galaxies within $30 \mathrm{Mpc}$, selected entirely based on their optical properties and spanning a wide range in absolute magnitude and stellar mass. For 61 objects we obtained new ACIS-S observations to a uniform limiting luminosity of $2.5 \times 10^{38} \mathrm{erg} \mathrm{s}^{-1}$, and for the remaining 42 objects we make use of high-quality archival coverage. The primary results of this paper are as follows:

1. The detection fraction of nuclear X-ray sources is 52/103. A handful of these may be contaminated by LMXBs; we estimate that the fraction of accreting SMBHs is $45 \% \pm 7 \%$. This provides a firm lower limit on the SMBH occupation fraction within field spheroids.

2. The ratio of X-ray to Eddington luminosities within the Field sample is in all cases low, from $\sim 10^{-4}$ to $10^{-8}$. This range is similar to that found in the AMUSE-Virgo study of cluster early types (G10). Independent of environment, $\mathrm{SMBH}$ accretion within local early types is generally highly sub-Eddington.

3. We quantify the correlation between nuclear X-ray luminosity and host stellar mass, finding a best-fit relation of $\left(\log L_{X}-38.4\right)=(-0.04 \pm 0.12)+(0.71 \pm 0.10) \times$ $\left(\log M_{\text {star }}-9.8\right)$. The calculated slope is incompatible with zero dependence and marginally indicative $(2.9 \sigma)$ of greater $L_{\mathrm{X}}$ per unit $M_{\text {star }}$ in lower-mass galaxies.

4. After scaling by black hole mass or accounting for the influence of stellar mass, typical values of $\log L_{X} / L_{\text {Edd }}$ or $\Delta L_{\mathrm{X}, \mathrm{M} *}$ of group members may be slightly lower than for isolated galaxies, and this newly identified trend appears to continue to cluster early types. If confirmed, a progressive decrease in relative X-ray luminosity with increasing richness mandates an environmental modulation of SMBH fueling.

Several complementary projects that build on this data set are underway. A direct comparison of the nuclear X-ray luminosity as a function of black hole mass within field and cluster galaxies, controlling for stellar mass, is discussed in Miller et al. (2012). An additional later work will present the catalog of offnuclear X-ray sources within the field sample and compare the distribution of ULXs with that found for Virgo. We are also currently obtaining high-resolution HST/ACS imaging of Field galaxies with $\mathrm{X}$-ray detections, to check for and characterize any nuclear star clusters.

We thank Kayhan Gültekin for sharing his Chandra data in advance of publication, we thank him and Marta Volonteri for useful conversations, and we thank an anonymous referee for constructive suggestions that improved this paper. T.T. acknowledges support from the NSF through CAREER award NSF0642621, and from the Packard Foundation through a Packard Research Fellowship. J.H.W. acknowledges support by the Basic Science Research Program through the National Research Foundation of Korea funded by the Ministry of Education, Science and Technology (2010-0021558). Support for this work was provided by NASA through Chandra Award Numbers 11620915 (AMUSE-Field) and 08900784 (AMUSE-Virgo) issued by the Chandra X-ray Observatory Center. This research has made use of the NASA/IPAC Extragalactic Database (NED), which is operated by the Jet Propulsion Laboratory, California Institute of Technology, under contract with NASA. We acknowledge the usage of the HyperLeda database.

\section{APPENDIX}

\section{NOTES ON SELECTED ARCHIVAL $L_{X}$ MEASUREMENTS}

$N G C 5846$. The X-ray morphology of this galaxy is complex, with a cavity to the northeast bounded by a ring, with similar 
structure also seen in $\mathrm{H} \alpha$ (Trinchieri \& Goudfrooij 2002). The optical center of the galaxy is near the radio core (Dunn et al. 2010) and corresponds to object 12 in the catalog of Trinchieri \& Goudfrooij (2002). Filho et al. (2004) find $L_{X}=$ $5.6 \times 10^{38} \mathrm{erg} \mathrm{s}^{-1}(0.5-10 \mathrm{keV})$. While the nucleus is identified by wavdetect in the full-band image, it is difficult to estimate the local (gas) background, and we do not find a significant detection in the hard-band image $\left(L_{\mathrm{X}}<9.5 \times 10^{38} \mathrm{erg} \mathrm{s}^{-1}\right)$. This is the primary galaxy of the largest group within the Field sample.

NGC 3923. Boroson et al. (2011) do not find an AGN component to be detectable in this galaxy, and we agree with this assessment. While wavdetect finds a potential nuclear source in the full-band image, the enclosing ellipse is substantially larger than the PSF, and the hard-band image shows complex extended emission, likely from several unresolved sources.

IC 1459. Fabbiano et al. (2003) find that the nuclear emission of this radio galaxy is well fit with an unabsorbed power law with $\Gamma=1.9$, and their $L_{\mathrm{X}}$ is consistent with our value of $10^{41} \mathrm{erg} \mathrm{s}^{-1}$; this source has the greatest X-ray luminosity in the Field sample.

$N G C$ 4636. Loewenstein et al. (2001) describe a "nearnuclear" source that we find to be coincident with the galactic center (offset $\sim 0$. 4 ; our applied astrometry correction of $00^{\prime \prime} 1$ is less than their $0{ }^{\prime \prime} 9$ ), and this source matches the location of the radio core (Dunn et al. 2010). As described in Dunn et al. (2010), this galaxy is 2.6 Mpc from M87 and shows disturbed $\mathrm{X}$-ray morphology.

NGC 1332. Humphrey \& Buote (2004) do not find evidence for a point-like central source and give a limit of $L_{\mathrm{X}}<$ $8.4 \times 10^{38} \mathrm{erg} \mathrm{s}^{-1}$. We measure a significant detection at the peak of the hard-band emission, which is concentrated at the nucleus.

NGC 5576 and NGC 2778. Gültekin et al. (2012) find somewhat greater (unabsorbed) $L_{X}$ values for these sources, although owing to limited counts the uncertainties are necessarily large. Their best-fit spectral models have steep power-law indices ( $\Gamma \sim 3.5$ and 4.6, respectively) and some intrinsic absorption, which differs significantly from the generic model we use to convert counts to fluxes.

$N G C$ 3384. From an early-short exposure, Boroson et al. (2011) quote a loose upper limit to the AGN component, whereas Zhang et al. (2009) do find an X-ray nucleus with $L_{\mathrm{X}}$ matching our value of $6.3 \times 10^{38} \mathrm{erg} \mathrm{s}^{-1}$; our measurement is based on a subsequent deeper exposure as detailed in Gültekin et al. (2012), who also find a similar $L_{X}$.

NGC 3115. Wong et al. (2011) describe the central emission as a "plateau" and give only an upper limit for the X-ray nucleus. Our $L_{\mathrm{X}}$ value is consistent with that given by Boroson et al. (2011), but it does appear possible that this is somewhat ( $\sim 50 \%$ or 0.2 dex) overestimated owing to at least one additional source crowding the nucleus.

$N G C$ 4278. Brassington et al. (2009) analyze six deep exposures (combined $458 \mathrm{ks}$ ) of this galaxy; the LINER nucleus is object 117 in their catalog. They find $L_{X}=2.0 \times 10^{40} \mathrm{erg} \mathrm{s}^{-1}$ (as do Boroson et al. 2011), which is $\sim 0.5$ dex greater than our value; 0.3 dex of the discrepancy is due to their greater distance (likely more accurate than our radial-velocity value from HyperLeda in this instance), and the remainder may reflect intrinsic variability: for the ObsID 7081 we use, Brassington et al. (2009) find $L_{X}$ lower by 0.2 dex than their overall exposureweighted mean.

$N G C$ 3377. The central source in this galaxy connects to an Eastward "bridge" of emission, which includes at least one additional source. Boroson et al. (2011) do not find an AGN component to be detectable, but Zhang et al. (2009) list $L_{\mathrm{X}}=3.9 \times 10^{38} \mathrm{erg} \mathrm{s}^{-1}(0.3-8 \mathrm{keV})$, in good agreement with our value.

$N G C$ 4627. This nearby ( $\sim 10.4 \mathrm{Mpc})$ galaxy is serendipitously present off-axis in a deep exposure targeting NGC 4631, but we do not detect it in our aperture photometry, and Zhang et al. (2009) also give only an upper limit. This source has the most stringent limit on X-ray luminosity $\left(L_{\mathrm{X}}<8.5 \times\right.$ $10^{36} \mathrm{erg} \mathrm{s}^{-1}$ ) within the Field sample.

\section{REFERENCES}

Acreman, D. M., Stevens, I. R., Ponman, T. J., \& Sakelliou, I. 2003, MNRAS, 341,1333

Baganoff, F. K., Bautz, M. W., Brandt, W. N., et al. 2001, Nature, 413, 45

Balmaverde, B., Baldi, R. D., \& Capetti, A. 2008, A\&A, 486, 119

Bauer, F. E., Alexander, D. M., Brandt, W. N., et al. 2004, AJ, 128, 2048

Begelman, M. C. 2010, MNRAS, 402, 673

Bekki, K., \& Graham, A. W. 2010, ApJ, 714, L313

Bell, E. F., \& de Jong, R. S. 2001, ApJ, 550, 212

Bell, E. F., McIntosh, D. H., Katz, N., \& Weinberg, M. D. 2003, ApJS, 149, 289

Bloom, J. S., Giannios, D., Metzger, B. D., et al. 2011, Science, 333, 203

Boroson, B., Kim, D.-W., \& Fabbiano, G. 2011, ApJ, 729, 12

Brassington, N. J., Fabbiano, G., Kim, D.-W., et al. 2009, ApJS, 181, 605

Brockamp, M., Baumgardt, H., \& Kroupa, P. 2011, MNRAS, 418, 1308

Brown, B. A., \& Bregman, J. N. 2000, ApJ, 539, 592

Brown, M. J. I., Brand, K., Dey, A., et al. 2006, ApJ, 638, 88

Buckley, J., \& James, I. 1979, Biometrics, 66, 429

Burrows, D. N., Kennea, J. A., Ghisellini, G., et al. 2011, Nature, 476, 421

Cannizzo, J. K., Lee, H. M., \& Goodman, J. 1990, ApJ, 351, 38

Cannizzo, J. K., Troja, E., \& Lodato, G. 2011, ApJ, 742, 32

Choi, Y.-Y., Woo, J.-H., \& Park, C. 2009, ApJ, 699, 1679

Ciotti, L., Ostriker, J. P., \& Proga, D. 2009, ApJ, 699, 89

Ciotti, L., Ostriker, J. P., \& Proga, D. 2010, ApJ, 717, 708

Côté, P., Blakeslee, J. P., Ferrarese, L., et al. 2004, ApJS, 153, 223

Croom, S. M., Richards, G. T., Shanks, T., et al. 2009, MNRAS, 399, 1755

Croton, D. J., Springel, V., White, S. D. M., et al. 2006, MNRAS, 365, 11

De Lucia, G., Springel, V., White, S. D. M., Croton, D., \& Kauffmann, G. 2006, MNRAS, 366, 499

Di Matteo, T., Quataert, E., Allen, S. W., Narayan, R., \& Fabian, A. C. 2000, MNRAS, 311, 507

Dunn, R. J. H., Allen, S. W., Taylor, G. B., et al. 2010, MNRAS, 404, 180

Eckart, M. E., Laird, E. S., Stern, D., et al. 2005, ApJS, 156, 35

Eliche-Moral, M. C., Prieto, M., Gallego, J., et al. 2010, A\&A, 519, A55

Fabbiano, G., Elvis, M., Markoff, S., et al. 2003, ApJ, 588, 175

Fabbiano, G., Kim, D.-W., Fragos, T., et al. 2006, ApJ, 650, 879

Ferrarese, L., Côté, P., Dalla Bontà, E., et al. 2006a, ApJ, 644, L21

Ferrarese, L., Côté, P., Jordán, A., et al. 2006b, ApJS, 164, 334

Ferrarese, L., \& Ford, H. 2005, Space Sci. Rev., 116, 523

Filho, M. E., Fraternali, F., Markoff, S., et al. 2004, A\&A, 418, 429

Fukugita, M., Ichikawa, T., Gunn, J. E., et al. 1996, AJ, 111, 1748

Gallo, E., Treu, T., Jacob, J., et al. 2008, ApJ, 680, 154

Gallo, E., Treu, T., Marshall, P. J., et al. 2010, ApJ, 714, 25

Gavazzi, G., Fumagalli, M., Cucciati, O., \& Boselli, A. 2010, A\&A, 517, A73 Gehrels, N. 1986, ApJ, 303, 336

Ghosh, K. K., Saripalli, L., Gandhi, P., et al. 2009, AJ, 137, 3263

Gilfanov, M. 2004, MNRAS, 349, 146

González-Martín, O., Masegosa, J., Márquez, I., \& Guainazzi, M. 2009, ApJ, 704,1570

Goulding, A. D., Alexander, D. M., Lehmer, B. D., \& Mullaney, J. R. 2010, MNRAS, 406, 597

Gnedin, O. Y. 2003, ApJ, 582, 141

Graham, A. W. 2008, ApJ, 680, 143

Graham, A. W., Onken, C. A., Athanassoula, E., \& Combes, F. 2011, MNRAS, 412, 2211

Graham, A. W., \& Spitler, L. R. 2009, MNRAS, 397, 2148

Greene, J. E., Peng, C. Y., Kim, M., et al. 2010, ApJ, 721, 26

Gültekin, K., Cackett, E. M., Miller, J. M., et al. 2012, ApJ, submitted

Gültekin, K., Richstone, D. O., Gebhardt, K., et al. 2009, ApJ, 698, 198

Hasinger, G., Miyaji, T., \& Schmidt, M. 2005, A\&A, 441, 417

Healey, S. E., Romani, R. W., Taylor, G. B., et al. 2007, ApJS, 171, 61

Heckman, T. M., Kauffmann, G., Brinchmann, J., et al. 2004, ApJ, 613, 109

Ho, L. C. 1999, ApJ, 516, 672 
Ho, L. C. 2008, ARA\&A, 46, 475

Ho, L. C., Filippenko, A. V., \& Sargent, W. L. W. 1997, ApJ, 487, 591

Hong, J., van den Berg, M., Schlegel, E. M., et al. 2005, ApJ, 635, 907

Hopkins, P. F., Hernquist, L., Martini, P., et al. 2005, ApJ, 625, L71

Hopkins, P. F., \& Quataert, E. 2011, MNRAS, 411, L61

Hopkins, P. F., Richards, G. T., \& Hernquist, L. 2007, ApJ, 654, 731

Humphrey, P. J., \& Buote, D. A. 2004, ApJ, 612, 848

Humphrey, P. J., \& Buote, D. A. 2010, MNRAS, 403, 2143

Irwin, J. A., Athey, A. E., \& Bregman, J. N. 2003, ApJ, 587, 356

Irwin, J. A., Bregman, J. N., \& Athey, A. E. 2004, ApJ, 601, L143

Jiang, L., Fan, X., Brandt, W. N., et al. 2010, Nature, 464, 380

Kang, X., van den Bosch, F. C., \& Pasquali, A. 2007, MNRAS, 381, 389

Kaplan, E. L., \& Meier, P. 1968, J. Am. Stat. Assoc., 53, 457

Kelly, B. C. 2007, ApJ, 665, 1489

Kelly, B. C., Vestergaard, M., Fan, X., et al. 2010, ApJ, 719, 1315

Kim, D.-W., \& Fabbiano, G. 2010, ApJ, 721, 1523

King, A. R., Davies, M. B., Ward, M. J., Fabbiano, G., \& Elvis, M. 2001, ApJ, 552, L109

Kormendy, J., Fisher, D. B., Cornell, M. E., \& Bender, R. 2009, ApJS, 182, 216

Kraft, R. P., Burrows, D. N., \& Nousek, J. A. 1991, ApJ, 374, 344

Kreckel, K., Platen, E., Aragón-Calvo, M. A., et al. 2011, AJ, 141, 4

Lamastra, A., Menci, N., Maiolino, R., Fiore, F., \& Merloni, A. 2010, MNRAS, 405, 29

Latta, R. P. 1981, J. Am. Stat. Assoc., 76, 713

Lauer, T. R., Tremaine, S., Richstone, D., \& Faber, S. M. 2007, ApJ, 670, 249

Lavalley, M., Isobe, T., \& Feigelson, E. 1992, in ASP Conf. Ser. 25, Astronomical Data Analysis Software and Systems I, ed. D. M. Worrall, C. Biemesderfer, \& J. Barnes (San Francisco, CA: ASP), 245

Levan, A. J., Tanvir, N. R., Cenko, S. B., et al. 2011, Science, 333, 199

Li, L.-X., Narayan, R., \& Menou, K. 2002, ApJ, 576, 753

Lin, D., Carrasco, E. R., Grupe, D., et al. 2011, ApJ, 738, 52

Liu, J. 2011, ApJS, 192, 10

Lodato, G., King, A. R., \& Pringle, J. E. 2009, MNRAS, 392, 332

Loewenstein, M., Mushotzky, R. F., Angelini, L., Arnaud, K. A., \& Quataert, E. 2001, ApJ, 555, L21

Luo, B., Brandt, W. N., Steffen, A. T., \& Bauer, F. E. 2008, ApJ, 674, 122

Makarov, D., \& Karachentsev, I. 2011, MNRAS, 412, 2498

Marulli, F., Bonoli, S., Branchini, E., Moscardini, L., \& Springel, V. 2008, MNRAS, 385, 1846

Mayer, L., Kazantzidis, S., Escala, A., \& Callegari, S. 2010, Nature, 466, 1082

Mei, S., Blakeslee, J. P., Côté, P., et al. 2007, ApJ, 655, 144

Merloni, A., \& Heinz, S. 2007, MNRAS, 381, 589

Merritt, D., \& Wang, J. 2005, ApJ, 621, L101

Miller, B., Gallo, E., Treu, T., \& Woo, J.-H. 2012, ApJ, 745, L13

Miller, J. M., \& Gültekin, K. 2011, ApJ, 738, L13

Moretti, A., Campana, S., Lazzati, D., \& Tagliaferri, G. 2003, ApJ, 588, 696
Nemmen, R. S., Storchi-Bergmann, T., Yuan, F., et al. 2006, ApJ, 643, 652 Netzer, H., Lira, P., Trakhtenbrot, B., Shemmer, O., \& Cury, I. 2007, ApJ, 671, 1256

Oosterloo, T., Morganti, R., Crocker, A., et al. 2010, MNRAS, 409, 500

O'Sullivan, E., Forbes, D. A., \& Ponman, T. J. 2001, MNRAS, 328, 461

Paturel, G., Petit, C., Prugniel, P., et al. 2003, A\&A, 412, 45

Pellegrini, S. 2005, ApJ, 624, 155

Pellegrini, S. 2010, ApJ, 717, 640

Pellegrini, S., Ciotti, L., \& Ostriker, J. P. 2012, ApJ, 744, 21

Rees, M. J. 1988, Nature, 333, 523

Schawinski, K., Urry, C. M., Virani, S., et al. 2010, ApJ, 711, 284

Schödel, R., Merritt, D., \& Eckart, A. 2009, A\&A, 502, 91

Schulze, A., \& Wisotzki, L. 2010, A\&A, 516, A87

Seth, A., Agüeros, M., Lee, D., \& Basu-Zych, A. 2008, ApJ, 678, 116

Shankar, F., Weinberg, D. H., \& Miralda-Escudé, J. 2009, ApJ, 690, 20

Shemmer, O., Brandt, W. N., Schneider, D. P., et al. 2006, ApJ, 644, 86

Shin, M.-S., Ostriker, J. P., \& Ciotti, L. 2012, ApJ, 745, 13

Sivakoff, G. R., Jordán, A., Sarazin, C. L., et al. 2007, ApJ, 660, 1246

Sivakoff, G. R., Sarazin, C. L., \& Jordán, A. 2005, ApJ, 624, L17

Soltan, A. 1982, MNRAS, 200, 115

Soria, R., Fabbiano, G., Graham, A. W., et al. 2006a, ApJ, 640, 126

Soria, R., Graham, A. W., Fabbiano, G., et al. 2006b, ApJ, 640, 143

Strubbe, L. E., \& Quataert, E. 2009, MNRAS, 400, 2070

Strubbe, L. E., \& Quataert, E. 2011, MNRAS, 415, 168

Thomas, D., Maraston, C., Bender, R., \& Mendes de Oliveira, C. 2005, ApJ, 621,673

Treister, E., Schawinski, K., Volonteri, M., Natarajan, P., \& Gawiser, E. 2011, Nature, 474, 356

Treu, T., Ellis, R. S., Kneib, J.-P., et al. 2003, ApJ, 591, 53

Treu, T., Woo, J.-H., Malkan, M. A., \& Blandford, R. D. 2007, ApJ, 667, 117

Trinchieri, G., \& Goudfrooij, P. 2002, A\&A, 386, 472

Véron-Cetty, M.-P., \& Véron, P. 2006, A\&A, 455, 773

Vestergaard, M., \& Osmer, P. S. 2009, ApJ, 699, 800

Volonteri, M. 2010, Nature, 466, 1049

Volonteri, M., Dotti, M., Campbell, D., \& Mateo, M. 2011, ApJ, 730, 145

Volonteri, M., \& Natarajan, P. 2009, MNRAS, 400, 1911

Volonteri, M., \& Rees, M. J. 2006, ApJ, 650, 669

Willott, C. J., Albert, L., Arzoumanian, D., et al. 2010, AJ, 140, 546

Wong, K.-W., Irwin, J. A., Yukita, M., et al. 2011, ApJ, 736, L23

Woo, J.-H., Treu, T., Barth, A. J., et al. 2010, ApJ, 716, 269

Woo, J.-H., Treu, T., Malkan, M. A., \& Blandford, R. D. 2008, ApJ, 681, 925

Yu, Q., \& Tremaine, S. 2002, MNRAS, 335, 965

Zhang, W. M., Soria, R., Zhang, S. N., Swartz, D. A., \& Liu, J. F. 2009, ApJ, 699, 281

Zhang, Z.-L., \& Xu, H.-G. 2004, Chin. J. Astron. Astrophys., 4, 221 